\title{
Active suspensions in thin films: nutrient uptake and swimmer motion
}

\author{
Ruth A. Lambert ${ }^{1}$, Francesco Picano ${ }^{1}$, Wim-Paul Breugem ${ }^{2}$ \\ and Luca Brandt ${ }^{1,3,}+$ \\ ${ }^{1}$ Linné Flow Centre, KTH Mechanics, SE-100 44, Stockholm, Sweden \\ ${ }^{2}$ Delft University of Technology, Laboratory for Aero and Hydrodynamics, Leeghwaterstraat 21, \\ NL-2628 CA Delft, The Netherlands \\ ${ }^{3}$ SeRC, Swedish e-Science Research Centre, KTH Mechanics, SE-100 44, Stockholm, Sweden
}

(Received 23 November 2012; revised 4 July 2013; accepted 31 August 2013)

\begin{abstract}
A numerical study of swimming particle motion and nutrient transport is conducted for a semidilute to dense suspension in a thin film. The steady squirmer model is used to represent the motion of living cells in suspension with the nutrient uptake by swimming particles modelled using a first-order kinetic equation representing the absorption process that occurs locally at the particle surface. An analysis of the dynamics of the neutral squirmers inside the film shows that the vertical motion is reduced significantly. The mean nutrient uptake for both isolated and populations of swimmers decreases for increasing swimming speeds when nutrient advection becomes relevant as less time is left for the nutrient to diffuse to the surface. This finding is in contrast to the case where the uptake is modelled by imposing a constant nutrient concentration at the cell surface and the mass flux results to be an increasing monotonic function of the swimming speed. In comparison to non-motile particles, the cell motion has a negligible influence on nutrient uptake at lower particle absorption rates since the process is rate limited. At higher absorption rates, the swimming motion results in a large increase in the nutrient uptake that is attributed to the movement of particles and increased mixing in the fluid. As the volume fraction of swimming particles increases, the squirmers consume slightly less nutrients and require more power for the same swimming motion. Despite this increase in energy consumption, the results clearly demonstrate that the gain in nutrient uptake make swimming a winning strategy for micro-organism survival also in relatively dense suspensions.
\end{abstract}

Key words: biological fluid dynamics, micro-organism dynamics, multiphase and particle-laden flows

\section{Introduction}

The aim of this study is to compute numerically the nutrient transport and uptake in a thin film populated by swimming micro-organisms when the fluid motion and the mass transfer of nutrients are generated by the particle swimming and the nutrient absorption at the particle surface. The study of active particle motion in a fluid 
has important applications for understanding living cellular organisms. These include species of bacteria, green algae and marine plant-like organisms such as phytoplankton. The supply of nutrients is important for the overall health of suspensions of living cellular organisms where the nutrients include dissolved gasses such as oxygen, proteins, organic compounds and small particles.

The motion of active particles in confined environments is commonly observed in many controlled laboratory settings, e.g. Petri dishes, droplets, thin soap films, or films in glass slides (Berke et al. 2008). In confined environments the swimming particle volume fraction $\phi$ can range from the semidilute to dense suspensions. Numerical simulations of such suspensions require therefore the capability of resolving multiple particle interactions. Prior numerical work of fully resolved flow focuses largely on active suspensions in unbounded domains with mass transport analysis limited to single swimming particles. In this study, the effect of swimming motion on nutrient uptake in thin films is explored by means of fully resolved simulations using an immersed boundary method (IBM) for finite-size particles combined with a soft-sphere collision model (Breugem 2012).

The behaviour of single micro-organisms and active suspensions has been studied in many instances (see e.g. Koch \& Subramanian 2011). However, only a few of the numerical studies of individual swimming particles, including biofilms, that provide insight into the behaviour of active suspensions include mass transfer and nutrient uptake (Magar, Goto \& Pedley 2003; Magar \& Pedley 2005; Michelin \& Lauga 2011; Taherzadeh, Picioreanu \& Horn 2012). In these studies, the concentration of nutrients in the fluid medium are typically treated as a passive scalar. In the case of individual swimming particles, Magar et al. (2003), Magar \& Pedley (2005) and Michelin \& Lauga (2011) use a widely adopted unsteady and steady swimming cell model first introduced by Lighthill (1952) and Blake (1971). In this model, referred to as the squirmer model, the swimming cell is assumed to be spherical in shape with an applied tangential surface velocity that represents the surface distortion of beating cilia. This generalization allows us to classify the swimming motion into three swimming modes (pushers, pullers and neutral swimmers), and is in good agreement with the swimming motion of several micro-organisms such as Parmecium and Opalina (Ishikawa, Locsei \& Pedley 2008). The simple neutral and steady model used here has been adopted in several investigations of processes related to the physics of swimming micro-organisms, such as locomotion in stratified (Doostmohammadi, Stocker \& Ardekani 2012) and viscoelastic fluids (Zhu et al. 2011; Zhu, Lauga \& Brandt 2012).

Owing to a wide range in the values of the nutrient mass diffusivities $D$ of typical nutrients, both convective and diffusive mass transport occur in the fluid (Musielak et al. 2009) while the fluid motion is dominated by viscous diffusion. In a study of a single swimming particle, Magar et al. (2003) and Magar \& Pedley (2005) show that the swimming motion of a single cell increases the mass flux at the surface of the particle in comparison with an inert translating sphere. The mass flux is quantified in terms of the Sherwood number, a dimensionless nutrient concentration gradient at the particle surface. The increase in the mass flux at the particle surface increases with larger swimming speeds or increasing Péclet numbers. The Péclet number $P e=U_{s} d / D$, where $d$ is the cell diameter and $U_{s}$ is the cell swimming speed, is the ratio of convective to diffusive mass transport in the fluid. For a free swimming particle the increase in the mass flux at the particle surface scales to $P e^{1 / 2}$ in comparison with $P e^{1 / 3}$ for an inert translating sphere. Similarly, Short et al. (2006) study molecular transport in suspensions of Volvocine green algae and demonstrate that the increase 
of uptake due to swimming permits cells of radius $r>10 \mu \mathrm{m}$ to overcome diffusion limitations and satisfy metabolic requirements. In an optimization study of the nutrient uptake and swimming mode of an individual swimming particle, Michelin \& Lauga (2011) show that the optimal swimming motion coincides with the optimal nutrient uptake for all Péclet numbers. All of these results are obtained assuming constant nutrient concentration at the particle surface. In Magar et al. (2003) and Magar \& Pedley (2005) a first-order nutrient uptake model is also included in an appendix. These authors perform asymptotic analysis in the limit of very small and very large Péclet numbers and show that the uptake decreases in this case. The first-order rate equation for nutrient uptake at the particle surface has been used in studies of single swimming particle motion, biofilms (Taherzadeh et al. 2012) and diatom chains (Musielak et al. 2009). This study is the first to adopt a similar simple nutrient uptake model and apply it to a suspension of swimming particles in an intermediate range of Péclet numbers.

The connection between swimming cellular organisms and enhanced mixing in the fluid is often discussed in terms of an effective fluid diffusivity that can be incorporated into continuum models. In a three-dimensional numerical study of swimming particle motion in unbounded flow, Ishikawa \& Pedley (2007a) and Ishikawa, Locsei \& Pedley (2010) show that the effective diffusivity in the fluid increases as the cellular volume fraction of the suspension increases, whereas the diffusivity of swimmers decreases. Underhill, Hernandez-Ortiz \& Graham (2008) found that the diffusivity is affected by the swimming particle mode, with pushers enhancing fluid diffusivity and pullers less. In experiments of swimming algal cells in thin films, Kurtuldu et al. (2011) showed that the reduced dimensionality of the thin film further enhances the diffusivity which increases rapidly with volume fraction and follows a Gaussian distribution at volume fractions above $7 \%$.

Various numerical studies have confirmed experimental observations of coherent jetlike and vortex-like structures in three-dimensional or unbounded domains (Saintillan \& Shelley 2007; Ishikawa et al. 2008; Evans et al. 2011, to cite a few). Swimming particles align with neighbouring particles as a consequence of near-field interactions resulting in organized large-scale motion (Ishikawa \& Pedley 2008; Lin, Thiffeault \& Childress 2011) that further enhances fluid mixing. Interestingly, the recent experiments by Ishikawa et al. (2011) show that in concentrated suspensions of swimming bacteria, this collective behaviour is energy efficient in the sense that a very small fraction of the dissipated energy from swimming is transferred to the large-scale motion that in turn produces very large gains in the mass transport in the fluid. In these investigations, however, no direct connection to nutrient uptake is discussed.

In a confined environment, the interactions of the swimming cells with the bounding surfaces affects the particle distribution and collective motion, something which has consequences for the nutrient transport and uptake. For suspensions in a bounded domain, the swimming cells accumulate near the surface, as observed for bacteria (Li et al. 2011) and up-swimming, bottom-heavy algae (Pedley \& Kessler 1992), two different cellular organisms with opposite swimming modes, one 'pushing' the other 'pulling'. Several experimental and theoretical studies of swimming particle interactions with surfaces show that the particle interaction with the wall can vary with particle size, swimming type and particle shape and the long time trajectory of a swimming particle moving near a surface is not easily predicted (Li et al. 2011; Berke et al. 2008; Spagnolie \& Lauga 2012; Zhu, Lauga \& Brandt 2013). While most of 
these studies were conducted for solid walls, the motion toward a stress free surface, such as an air and water interface, show some similarities.

Finally we note that active suspensions in two or three dimensions have relevant differences. In two dimensions, for example, the far-field effects of swimming motion extend farther as the velocity decays as $1 / r$ rather than $1 / r^{2}$ as in three dimensions. Hernandez-Ortiz, Stoltz \& Graham (2005) found that the tendency of particles to accumulate near thin film surfaces occurs at lower volume fractions. As the volume fraction increases, large-scale coherent motion occurs which disrupts the localization of particles near the surface, eventually mixing the particles across the fluid layer. Ishikawa \& Pedley (2008) conducted a numerical study of swimming particle motion in monolayers in which the size of the collective motion is in agreement with the experimental observations of the size of swirls in a bacterial bath (Wu \& Libchaber 2000). The collective motion in confined domains is several particle radii larger than the size of cohesive motion observed in unbounded domains (Ishikawa et al. 2008).

The objective of this study is to analyse the mass transport properties of an active suspension in a thin film and the effect of the cell nutrient absorption rate on the mean uptake by exploring the Sherwood-Péclet and the Sherwood-Damköhler number relationships where the Damköhler number is a dimensionless nutrient uptake rate. These relationships have not been explored for multiple swimming particles or for swimming particles in confined environments.

\section{Governing equations}

The scaling of the governing equations is based upon the characteristic variables for swimming cells in flow dominated by viscous diffusion. The characteristic length is the diameter, $d$, of the swimming particle and the characteristic velocity is the swimming speed, $U_{s}$, of a solitary swimmer in an unbounded domain. The characteristic time is the viscous diffusion time $d^{2} / v$ where $v$ is the viscosity of the fluid. The characteristic stress is a viscous stress, $\tau=\rho_{f} \nu U_{s} / d$, where $\rho_{f}$ is the density of the fluid. Using these scaling arguments, five dimensionless variables appear in the governing equations. The characteristic flow parameters are the Reynolds number $R e$, the Péclet number $P e$, the Schmidt number $S c=P e / R e=v / D$, which is the ratio of the nutrient diffusion time to the viscous diffusion time, and the solid particle to fluid density ratio, $\alpha=\rho_{p} / \rho_{f}$ where $\rho_{p}$ is the density of the solid. In addition, in our problem we examine variations of the Damköhler number $D a=k d^{2} / D$, where $k$ is a first-order reaction rate used in the nutrient absorption model. $D a$ is the ratio of the diffusion time and the nutrient uptake time.

It is important to note that when the viscous diffusion time is used as the characteristic time, the unsteady terms in the governing equations are retained. The retention of the time variation in fluid and particle motion permits a general solution that collapses to the steady solution and resolves time-dependent phenomena that might occur at the same time scale as the viscous diffusion. For swimming microorganisms ranging in diameter from $d=1$ to $100 \mu \mathrm{m}$ the Reynolds number can range between $R e=10^{-5}$ and $10^{-1}$ (Magar et al. 2003). In this low-Reynolds-number flow regime, convection in the fluid is negligible and the unsteady Stokes' equations for fluid flow apply. The diffusivity of nutrients such as oxygen and proteins in water can range from $10^{-11}$ to $10^{-7}$ resulting in a range of $P e$ values from $10^{-3}$ to $10^{2}$ (Magar et al. 2003). For the given range of nutrient diffusivities, a lower limit for the Schmidt number, which governs the mass diffusion time with respect to the diffusion time in 


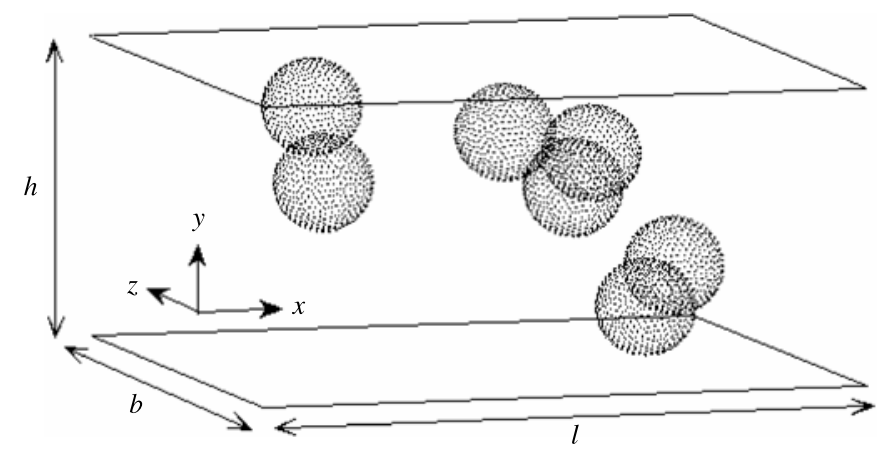

FIGURE 1. Diagram of the thin film with swimming particles represented by Lagrangian grid points.

the mass transport equation is $S c \simeq 100$. At the scale of swimming micro-organisms, both convective and diffusive mass transport are therefore relevant.

\subsection{Eulerian equations for fluid flow and mass transfer}

The scaling of the momentum equations for the fluid results in a dimensionless form where the Reynolds number only appears in front of the convective term. Since the Reynolds number is much less than unity, the convective term is neglected in the numerical implementation solving only the unsteady Stokes' equations. The dimensionless equations for the fluid are expressed as

$$
\begin{gathered}
\frac{\partial \boldsymbol{u}_{f}}{\partial t}=-\nabla p+\nabla^{2} \boldsymbol{u}_{f}+\boldsymbol{f}_{p}, \\
\nabla \cdot \boldsymbol{u}_{f}=0
\end{gathered}
$$

where $\boldsymbol{u}_{f}$ is the fluid velocity and $\boldsymbol{f}_{p}$ is the force resulting from the fluid and solid particle interactions.

The thin film is bounded by two open surfaces, representing for example an air and water interface, that extend in the horizontal $x$ and $z$ directions while the film thickness lies vertically in the $y$ direction as shown in figure 1. It is assumed that the surface tension of the interface is high enough such that the swimming particles are unable to break through the surface, resulting in a zero normal velocity component. For the tangential velocity components, the boundary condition is a homogenous Neumann boundary condition. The equations at the fluid boundary, $\partial \Omega_{f}$, are

$$
\begin{array}{ll}
\frac{\partial \boldsymbol{u}_{f}}{\partial \boldsymbol{x}} \cdot \boldsymbol{n}=0 & \text { with } \boldsymbol{x} \in \partial \Omega_{f} \\
\boldsymbol{u}_{f} \cdot \boldsymbol{n}=0 \quad \text { with } \boldsymbol{x} \in \partial \Omega_{f} .
\end{array}
$$

Periodic boundary conditions are implemented in the $x$ and $z$ directions.

Owing to the broad range in the Péclet number, both convective and diffusive mass transport play a role in nutrient uptake. The concentration of nutrients in the fluid is represented by a passive scalar, $c_{f}$. The dimensionless mass transport equation is expressed as

$$
\frac{\partial c_{f}}{\partial t}+\frac{P e}{S c} \boldsymbol{u}_{f} \cdot \nabla c_{f}=\frac{1}{S c} \nabla^{2} c_{f}+s,
$$


where $s$ is a source term representing the absorption of nutrients at the swimming particle surface. At an initial time, the nutrient concentration in the thin film is saturated. In our model the nutrients are replenished at the open surfaces which reflects the absorption of gases into the thin film or a steady nutrient supply from an external source. Periodic boundary conditions are maintained in the $x$ and $z$ directions. The initial conditions and boundary conditions are

$$
\begin{array}{ll}
c_{f}=1 & \text { at } t=0 ; \\
c_{f}=1 & \text { for } \boldsymbol{x} \in \partial \Omega_{f} .
\end{array}
$$

\subsection{The nutrient absorption model}

During the process of nutrient uptake by the swimming particles, the nutrients diffuse toward the particle surface and are absorbed into the cell in an enzymatic process (Musielak et al. 2009). Simple first-order kinetics, such as Minod kinetic equation, with and without a saturation concentration (Magar et al. 2003; Musielak et al. 2009; Taherzadeh et al. 2012) have been used in numerical models of nutrient absorption. In this study, the nutrient uptake at the cell surface is a volumetric absorption process that is characterized by a simple first-order kinetic equation without a saturation concentration. The nutrient concentration at the particle surface $c_{s}$ is absorbed at a rate described by the reaction rate, $k$. The absorbtion rate, $s$, is expressed in dimensionless form as

$$
s=-\frac{D a}{S c} c_{s} \delta\left(\boldsymbol{x}-\boldsymbol{x}_{s}\right) \quad \text { with } \boldsymbol{x}_{s} \in \partial \Omega_{p}
$$

The absorption rate, $s$, is the source term in (2.5) and is applied at the particle interface using the regularized delta function, $\delta$.

Further examination of the mass transport equation (2.5) shows that the first term on the right-hand side represents the diffusive transport of nutrients toward the surface while the second term represents the removal of the nutrients from the thin film during absorption into the solid particle.

By solving the mass transport equation and taking into account (2.8), the relationship between the mass flux at the surface of the particle and the absorption rate is

$$
J_{p} \equiv \frac{1}{S c} \oint_{S} \nabla c \cdot \boldsymbol{n} \mathrm{d} S=-\int_{V} s \mathrm{~d} V,
$$

where $S$ is the surface area of the particle and $V$ is the volume of the domain. The dimensionless mass flux is defined by the Sherwood number, $S h$, which is the ratio between the total mass flux and the diffusive mass flux. For a spherical particle the relationship between the Sherwood number and the mass flux at the particle surface, $J_{p}$, as defined in (2.9) is $S h=2 J_{p} / J_{R}$ where the reference flux $J_{R}$ is the diffusive flux for a stationary sphere in an unbounded domain. The diffusive flux for a spherical particle is $2 \pi d c_{\infty} / S c$, where $c_{\infty}=1$.

\subsection{Swimming particle model}

The swimming micro-organism is represented by an idealized model swimmer, where the propulsion mechanism is given by a steady tangential velocity applied at the particle surface representing the surface distortions of beating cilia or flagella. This so-called envelope model, first proposed by Lighthill (1952) and extended by Blake (1971), is also referred to as the steady squirmer model. The dimensionless tangential 
velocity, $u_{\theta}$, is a function of the polar angle, $\theta$, that is defined from the axis positioned through the front of the swimming particle. The surface velocity is usually described by the following kinematic condition

$$
u_{\theta}=\sin \theta(1+\beta \cos \theta),
$$

where the ratio of the squirmer modes is defined as $\beta=B_{2} / B_{1} ; B_{1}$ is the first squirmer mode coefficient that gives the far flow field and $B_{2}$ is the second squirmer mode or stresslet that defines the near-flow field. The ratio of the squirmer modes, $\beta$, governs the position and size of the flow recirculation regions and its value defines three swimming types, pushers, pullers and neutral swimmers. The surface velocity is normalized by the swimming speed of a solitary squirmer in an unbounded domain, which is equal to $U_{s}=2 / 3 B_{1}$ for all swimming types. In the present numerical analysis, the collective behaviour and mass transport of active suspensions of neutral swimmers, $\beta=0$, are considered. The analysis of the differences due to the nature of the near field interactions in suspensions of different swimming types are left as future work.

\subsection{Lagrangian equations for swimming particles}

The velocity at the solid surface, $\boldsymbol{u}_{p}$, is a combination of the translational velocity of the swimming particle, the angular velocity due to particle rotation and the prescribed tangential surface velocity that represents the propulsive swimming mechanism of moving surface cilia following the squirmer model. The equation for the surface velocity is expressed as

$$
\boldsymbol{u}_{p}=\boldsymbol{u}_{s}+\boldsymbol{\omega} \times \boldsymbol{r}+u_{\theta} \boldsymbol{e}_{\theta},
$$

where $\boldsymbol{u}_{s}$ is the translational or swimming velocity and $\boldsymbol{\omega}$ is the angular velocity of the particle.

The translational and angular velocities are determined from the Newton-Euler equations of motion for solid spheres. The equation for the particle swimming velocity is expressed in dimensionless form as

$$
\alpha V_{p} \frac{\mathrm{d} \boldsymbol{u}_{s}}{\mathrm{~d} t}=\oint_{S} \boldsymbol{\sigma} \cdot \boldsymbol{n} \mathrm{d} S+(\alpha-1) V_{p} F r \boldsymbol{e}_{y},
$$

where $V_{p}$ is the particle volume, and the fluid stress is $\sigma=-p \boldsymbol{I}+2 \boldsymbol{D}$, and the deformation tensor is $\boldsymbol{D}=1 / 2\left(\boldsymbol{\nabla} \boldsymbol{u}_{f}+\nabla \boldsymbol{u}_{f}^{\mathrm{T}}\right)$. The second term on the right-hand side of the equation represents the buoyancy force acting on the particle where the Froude number is $F r=g d^{2} / U_{s} v$. In the numerical analysis the motion of neutrally buoyant particles with $\alpha=1$ is considered since the settling velocity for swimming cells is typically smaller than the swimming speed. For a relative density difference of few per cent, the settling velocity is of the order of micrometres per second, thus at least one order of magnitude lower than micro-organisms swimming speeds.

The angular momentum equation in dimensionless form is expressed as

$$
\bar{I}_{p} \frac{\mathrm{d} \boldsymbol{\omega}}{\mathrm{d} t}=\oint_{S} \boldsymbol{r} \times \boldsymbol{\sigma} \cdot \boldsymbol{n} \mathrm{d} S,
$$

where $\boldsymbol{r}$ is the radial distance and $\bar{I}_{p}$ is the dimensionless moment of inertia for a sphere, $\alpha V_{p} / d^{3} / 10$. For bottom-heavy swimming cells with a centre of mass that is offset from the centre of volume, an additional gravitational term appears on the right-hand side of (2.13) orienting the swimming motion contrary to the direction of 
gravity. Studies of diffusive behaviour and coherent structures in active suspensions for bottom-heavy swimming particles have been conducted and are relevant in applications involving pullers such as green algae (Ishikawa \& Pedley 2007b; Ishikawa et al. 2008). A prior study also investigates the motion of bottom-heavy swimmers confined to motion in two dimensions, showing the development of lateral wavy band formation (Ishikawa \& Pedley 2008). In this study, bottom-heaviness is not considered.

The equations for particle motion used in the IBM model replace the surface integral for the stress with the volumetric integral of the fluid and solid interaction force, $f_{p}$, and a corrective force which accounts for the inertia of the fictitious fluid within the particle volume. The equations also include the lubrication force correction for near-field particle and surface interactions and the repulsive force for particle collisions with neighbouring particles or planar surfaces, see the Appendix for more details. The form of the Newton-Euler equations (2.12) and (2.13) used in the IBM method are expressed as

$$
\begin{array}{r}
\alpha \frac{\mathrm{d} \boldsymbol{u}_{s}}{\mathrm{~d} t}=-\frac{1}{V_{p}} \int_{V_{l}} \boldsymbol{f}_{p} \mathrm{~d} V_{l}+\frac{1}{V_{p}} \frac{\mathrm{d}}{\mathrm{d} t} \int_{V_{p}} \boldsymbol{u}_{f} \mathrm{~d} V_{p}+(\alpha-1) \text { Fr } \boldsymbol{e}_{y}+\boldsymbol{f}_{l c}+\boldsymbol{f}_{r} \\
\frac{\bar{I}_{p}}{V_{p}} \frac{\mathrm{d} \boldsymbol{\omega}}{\mathrm{d} t}=-\frac{1}{V_{p}} \int_{V_{l}} \boldsymbol{r} \times \boldsymbol{f}_{p} \mathrm{~d} V_{l}+\frac{1}{V_{p}} \frac{\mathrm{d}}{\mathrm{d} t} \int_{V_{p}} \boldsymbol{r} \times \boldsymbol{u}_{f} \mathrm{~d} V_{p},
\end{array}
$$

where $\boldsymbol{f}_{l c}$ is the corrective lubrication force and $\boldsymbol{f}_{r}$ is the repulsive force. In this work, the density ratio is $\alpha=1$ and buoyancy effects are neglected. The volumetric integral for the fluid and solid interaction force in both (2.14) and (2.15) is taken in the thin volume $V_{l}$ surrounding the Lagrangian grid points at the particle surface.

\section{Numerical method}

The motion of swimming cells in a thin film is simulated with a computationally efficient IBM for the coupling of finite-sized moving particles with the surrounding fluid flow. The original numerical model, developed by Uhlmann (2005) and expanded by Breugem (2012), was designed to model the interactions of multiple inert spheres with second-order accuracy. The near-field interactions and collisions between neighbouring particles and solid surfaces are modelled using both a corrective lubrication force and the soft-sphere collision model. In particular, the tangential lubrication force and the lubrication torque are neglected and only the normal component of the force is imposed. Indeed, the normal component of the lubrication forces is of order $1 / \epsilon$, whereas the tangential component is of order $\log \epsilon$, with $\epsilon$ the gap width. This is based on no-slip conditions of rigid spheres. The interactions between neighbouring particles and surfaces have been validated for the settling of a particle toward a wall and the motion of two spheres in close proximity (see Appendix and Breugem 2010). Note however that for the volume fractions investigated here, simulations without lubrication correction do not show any appreciable difference.

In the IBM method, the governing equations (2.1), (2.2) and (2.5) are solved throughout the entire domain on an Eulerian background grid. The spherical particle interface is defined by Lagrangian grid points that translate over the Eulerian grid as shown in figure 1 where we display the spherical particles, defined by the Lagrangian grid points, in the thin film. The fluid and solid equations are coupled using a force distribution around the particle interface and interpolation using a regularized delta function is used to transmit the fluid and solid interactions between the Lagrangian and Eulerian grids. When a particle approaches a surface or neighbouring particle 
within a small distance $\epsilon$, the lubrication force in the thin layer between the surfaces is calculated and applied in the particle equation of motion. During collision, the soft-sphere collision model is implemented. In the soft-sphere collision model, a small overlap between the particle and the contacting surface is permitted before a repulsive force is applied in the equations of motion for the colliding particle or particles. The collisions between multiple particles are considered and the numerical model is not limited to only two particles. The IBM method is therefore designed to resolve the fluid and solid interactions of dense suspensions and is not limited to the semidilute regime. Further details of the IBM method can be found in the Appendix and in Breugem (2010, 2012).

The fluid and solid interaction force, $f_{p}$, in (2.1) and (2.14) is calculated on the solid Lagrangian grid points and distributed onto the Eulerian background grid after a series of interpolations using a regularized Dirac delta function, $\delta_{d}$. Uppercase letters are used to represent variables defined on the Lagrangian grid points, $l$, and lowercase letters represent variables defined on the Cartesian grid points, ijk. The calculation of the force distribution on the Eulerian grid around the particle interface can be summarized as follows (Breugem 2012),

$$
\begin{gathered}
\boldsymbol{U}_{f}^{*}=\sum \boldsymbol{u}_{f}^{*} \delta_{d}\left(\boldsymbol{x}-\boldsymbol{X}^{n}\right) \Delta x \Delta y \Delta z, \\
\boldsymbol{F}_{p}=\frac{1}{\Delta t}\left(\boldsymbol{U}_{p}^{n}-\boldsymbol{U}_{f}^{*}\right), \\
\boldsymbol{f}_{p}=\sum \boldsymbol{F}_{p} \delta_{p}\left(\boldsymbol{x}-\boldsymbol{X}^{n}\right) \Delta V_{l},
\end{gathered}
$$

where $\boldsymbol{u}_{f}^{*}$ is the provisional velocity obtained from the used pressure-correction scheme and $\Delta V_{l}$ has been introduced previously as the thin volume surrounding the Lagrangian grid points at the particle surface. The summations in (3.1) and (3.3) are taken over the Eulerian and Lagrangian grid points, respectively. The particle velocity, $\boldsymbol{U}_{p}$, is calculated on the Lagrangian grid points by solving the following particle equations of motion, (2.10), (2.11), (2.14) and (2.15), in sequential order.

In the numerical implementation, the mass transport equation (2.5) is solved using the same numerical approach as the fluid equations. Explicit time integration is conducted using a third-order Runge-Kutta scheme while the diffusive term is solved using the semi-implicit Crank-Nicolson scheme. In the iterative process, the nutrient concentration field is corrected to account for the source term $s$. Following the same procedure for uppercase and lowercase variables used to calculate the fluid and solid interaction force, the concentration at the surface, $c_{s}$, and the absorption rate at the interface defined by the source term $s$ are calculated at a new time step $t^{n+1}$ using a provisional concentration $\boldsymbol{c}_{f}^{*}$ as

$$
\begin{gathered}
C_{f}^{*}=\sum c_{f}^{*} \delta_{d}\left(\boldsymbol{x}-\boldsymbol{X}^{n}\right) \Delta x \Delta y \Delta z, \\
C_{s}^{n+1}=C_{f}^{*}-\frac{D a}{S c} \frac{\Delta t}{2}\left(C_{f}^{*}+C_{s}^{n+1}\right), \\
S^{*}=\frac{1}{\Delta t}\left(C_{s}^{n+1}-C_{f}^{*}\right), \\
c_{f}^{n+1}=c_{f}^{*}+\Delta t s^{*} .
\end{gathered}
$$

The mass flux, $J_{p}$, given in (2.9) is calculated as

$$
J_{p}=-\sum S^{n+1} \Delta V_{l} .
$$



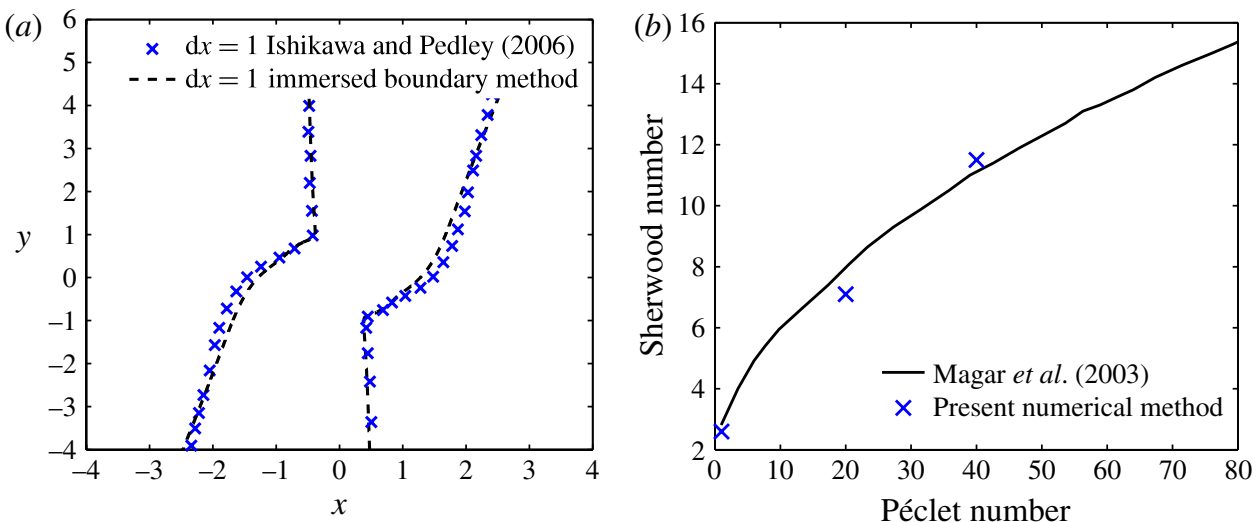

FIgURE 2. (Colour online) (a) Trajectories of squirmers with $\beta=5$ and initial distance $\mathrm{d} x=1$ in the transversal dimension. Comparison with Ishikawa, Simmonds \& Pedley (2006). (b) A comparison of the Sherwood and Péclet numbers for a single swimming particle in an unbounded domain with $\beta=1$ and a constant nutrient concentration at the particle surface. The Péclet number in the comparison is based on the particle radius, $r$.

\subsection{Numerical validation}

A grid size convergence test of the swimming speed $U_{s}$ of a single swimming particle in an unbounded domain was conducted for four different grid spacings $\Delta x$ of the background grid. In the test 16, 24, 32 and 36 grid points for cell diameter $d / \Delta x$ were used. The exact value of the swimming velocity is $U_{s}=1$. From the data, numerical implementation of the model swimmer is first-order accurate with an exponent of 1.07. Richardson extrapolation of the swimming speeds show that the results converge to a swimmer velocity of $U_{s}=1$ within four decimals for infinite resolution. In the numerical simulations, a grid spacing of $d / \Delta x=24$ is used producing an error in the swimming speed of $6.7 \%$. Although the error due to the numerical implementation results in a flow field that is less accurate than the Stokes' flow field solutions used to analyse dilute suspensions, this is deemed sufficient for our purposes.

The resulting error in the swimming particle velocity can be attributed to the high resolution required to resolve the flow field surrounding the swimming particle. Observations of the flow field cross-section show recirculation cells within the swimming particle with cores close to the edges of the sphere. At lower resolution, these flow features are not fully captured leading to errors in the interpolation of the velocity at the fluid and solid interface. The effect of the grid resolution on the accuracy of the particle swimming speeds were reported in the numerical work by Downton \& Stark (2009) who studied the motion of a solitary swimming particle in unbounded flow using a finite-volume flow solver. In the numerical study, errors as large as $15 \%$ for a grid spacing of $d / \Delta x=24$ were reported. A comparison with the work of Magar et al. (2003) and Ishikawa et al. (2006) is shown in figure 2. In figure 2(a) we report the trajectories of squirmers with initial distance $\mathrm{d} x=1$ in the transversal dimension, whereas the Sherwood and Péclet numbers for a single swimming particle in an unbounded domain for a constant nutrient concentration applied to the particle surface is displayed in figure $2(b)$. Note that in our simulations a finite domain is used with open boundary conditions for the velocity field and Dirichlet boundary conditions for the nutrient concentration in the $y$ direction. In the $x$ 
and $z$ directions the boundary conditions are periodic. More details and validations of the present method are provided in the Appendix.

\section{Results and discussion}

The motion of swimming particles in a thin film is considered in the absence of background flow for a semidilute to dense suspension. Three volume fractions of suspended particles are considered in the analysis, $\phi=0.078,0.16$ and 0.24 , corresponding to 16,32 and 50 squirmers in addition to the case of only one squirmer inside the film. The thickness of the film is $h=3 d$. The horizontal length, $l$, and width, $b$, of the numerical domain is $l=b=6 d$ as shown in figure 1 . The effect of the swimming particle motion on the mass transfer in the thin film is investigated for changes in the absorbtion rate at the particle surface $D a$, and the swimming Péclet number $\mathrm{Pe}$. In the analysis, changes in $\mathrm{Pe}$ reflect changes in the particle swimming speed, $U_{s}$. The Reynolds number $R e$ also changes with swimming speed but remains much less than one in real suspensions and therefore Stokes flow is considered here. The fluid properties, such as the viscosity, $v$, and the mass diffusivity, $D$, remain constant throughout the analysis (constant $S c$ ). To quantify the gain provided by swimming, the results for finite $P e$, swimming particles, are compared with the results of the diffusion limited case represented by a stationary array of fixed spheres of the same volume fraction, $\phi$, with the same nutrient absorption rate applied at the particle surface. The static array of particles is obtained for $\phi=0.078,0.16$ and 0.24 by a crystal configuration with 4, 4 and 5 rows aligned with the free-slip wall and 1, 2 and 2 columns. Each row contains equally spaced particles of 4, 4 and 5 particles per row, respectively. The vertical profiles of the static array are shown in figure 4.

Snapshots of the nutrient concentration contours in the thin film for three different absorption rates at the particle surfaces are shown in figure 3 for $\phi=0.16$ and $P e=100$. The swimming particles are initially positioned in an equidistant array with random orientations. The simulation times are long enough so that the results obtained after an initial time interval are no longer influenced by the initial conditions. The time to reach quasi-steady state in the solution of the mass transport equation is slightly longer than the time to reach dynamical equilibrium for the range in $P e$ values considered as shown in figure 9.

In the analysis, the effect of swimming motion on the mass transfer in the thin film is determined by analysing spatial and temporal averages of both the particle and fluid variables, as in the numerical work of Yeo \& Maxey (2010) among others. The mean or average values are calculated from an initial time $t=t_{o}$ over a large time interval, $T$, in the statistical steady-state regime. The mean value of the fluid variables are calculated using a Heaviside function $H(x, y, z)$ which has a value of $H=0$ in the particle domain and a value of $H=1$ in the fluid domain,

$$
\langle A\rangle_{f}(y)=\frac{\int_{t_{o}}^{T} \int_{S} H(x, y, z, t) A(x, y, z, t) \mathrm{d} x \mathrm{~d} z \mathrm{~d} t}{\int_{t_{o}}^{T} \int_{S} H(x, y, z, t) \mathrm{d} x \mathrm{~d} z \mathrm{~d} t} .
$$

\subsection{Collective particle behaviour}

The vertical profile of the normalized particle volume fraction along the cross-section is displayed in figure $4(a-c)$ for volume fractions of $\phi=0.078,0.16$ and 0.24 . The particle volume fraction, $\phi(y)$, shown in the figure is the summation of the total 

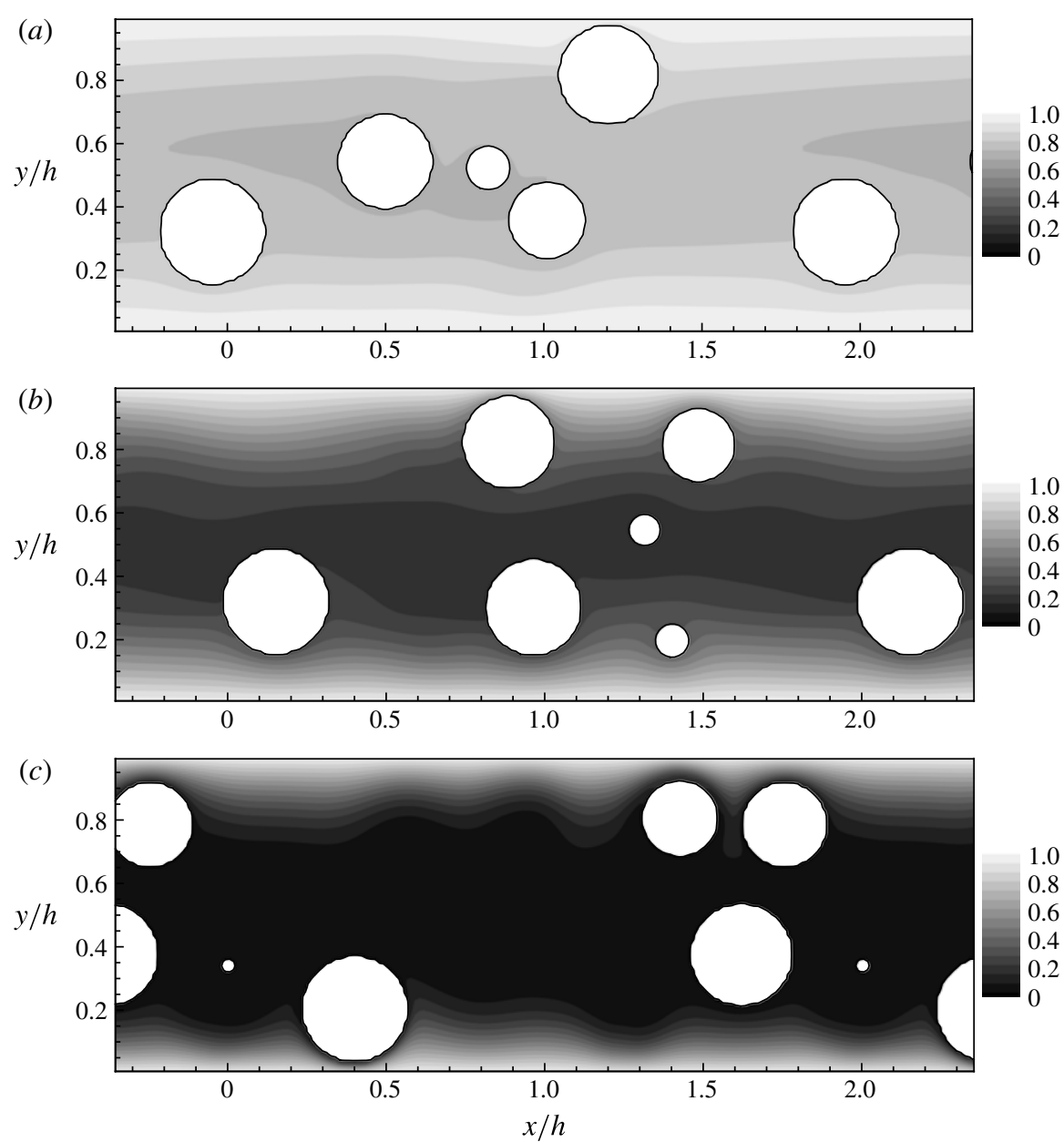

FIGURE 3. Nutrient concentration in an active suspension for absorption rates of $(a)$ $D a=10$, (b) $D a=100$ and (c) $D a=1000$ with $P e=100$ and $\phi=0.16$ in a plane orthogonal to the film boundaries. The concentration contours are taken after a long time interval at $t=240$ where white represents $c_{f}=1$ and black represents $c_{f}=0$. The particles move in and out of the visual frame of reference.

particle volume fraction along a horizontal cross-section, $b \times l$, with a thickness of $\Delta y$ and is normalized by the total volume fraction, $\phi$. For $\phi=0.078$ in figure $4(a)$, the particles form two layers in the thin film with a gap region along the centreline. For $\phi=0.16$ in figure $4(b)$, the particles begin to occupy the region in the centreline, with a larger accumulation closer to the film surface. As the volume fraction increases to $\phi=0.24$ (figure $4 c$ ), the squirmers become evenly distributed into three layers and the preferential accumulation of particles closer to the surface has disappeared. The plots also display the local concentration for a uniformly distributed stationary array of the same volume fraction (dashed line).

The particle trajectories in the thin film show the suppression of vertical particle motion after an initial transient phase whose average duration shows no variation with the volume fractions considered in this study. During an initial time interval, 

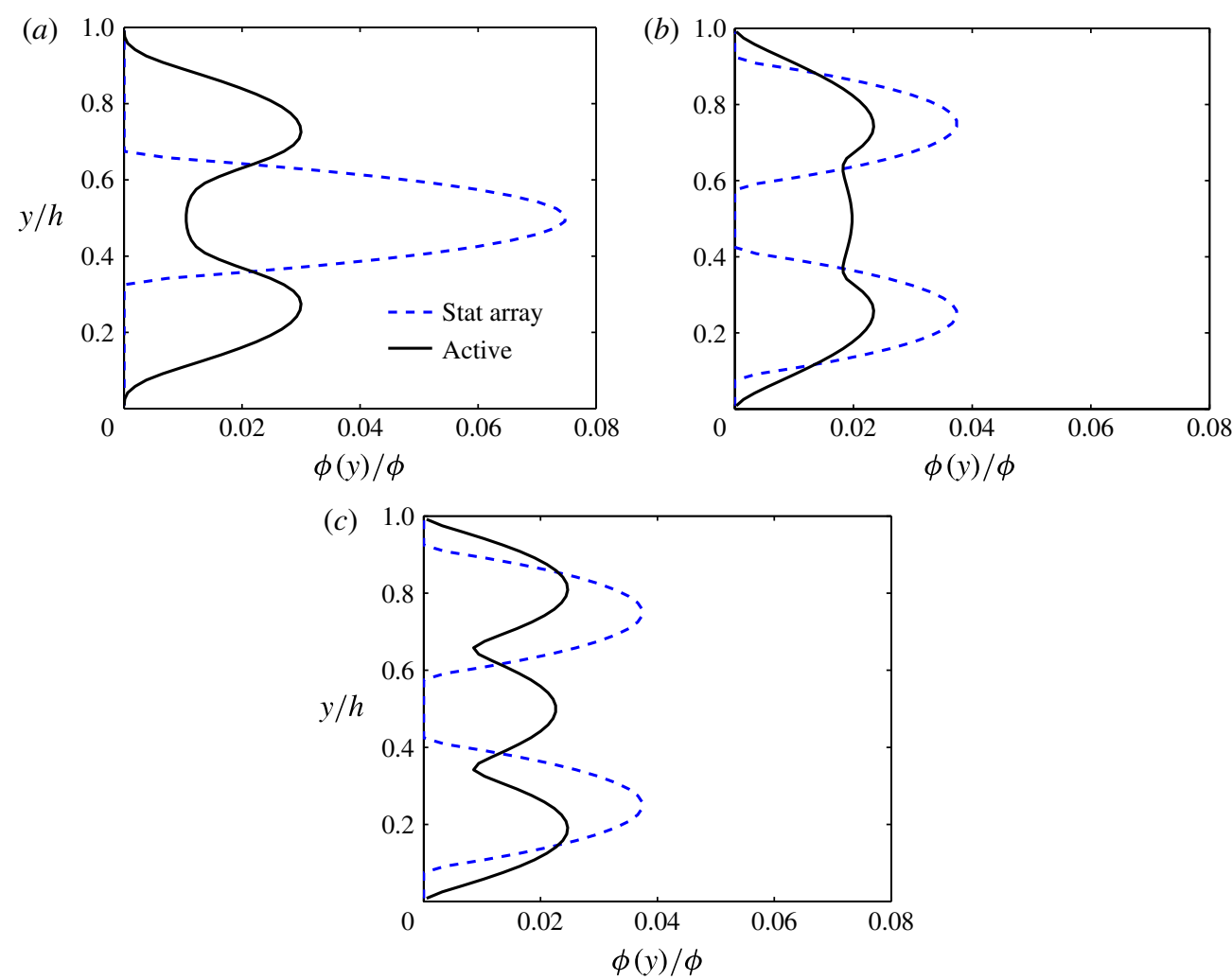

FIGURE 4. (Colour online) Vertical profile of the normalized volume fraction in an active suspension and a stationary array for $(a) \phi=0.078,(b) \phi=0.16$ and $(c) \phi=0.24$.

the particles move frequently between both surfaces. As the particles approach the surface they rotate and reorient themselves, swimming away at an angle that is almost equal to the approaching angle. The approaching particles may collide with the surface depending upon the approach angle and alterations to the trajectory by neighbouring particle interactions. After quasi-equilibrium is reached, which on average is the duration of 5-10 collisions with the surfaces, the particle paths show an increase in horizontal alignment and the mentioned reduction in the vertical motion. The swimming particles have an overall tendency to accumulate in two or three bands, three being the maximum number possible for a thin film of thickness $h=3 d$.

The vertical profiles of the fluid root-mean-squared (r.m.s.) velocity components in the planar and vertical directions are shown in figure 5 for the three different volume fractions under investigations. The results show that the overall fluid r.m.s. velocity increases with volume fraction. The ratio between the planar r.m.s. velocity, $u_{f, r m s}$, and the vertical r.m.s. velocity, $v_{f, r m s}$, also increases with the volume fraction, being larger than 2 for $\phi=0.24$. This reflects the suppression of the vertical particle motion in the film layer. This effect is even more apparent in the vertical profiles of the particle r.m.s. velocities shown in figure $6(a)$. The values of the particle r.m.s. velocities show that the vertical component is much less than one while the planar component is around unity, which indicates that particles are preferentially oriented parallel to the film boundaries. 

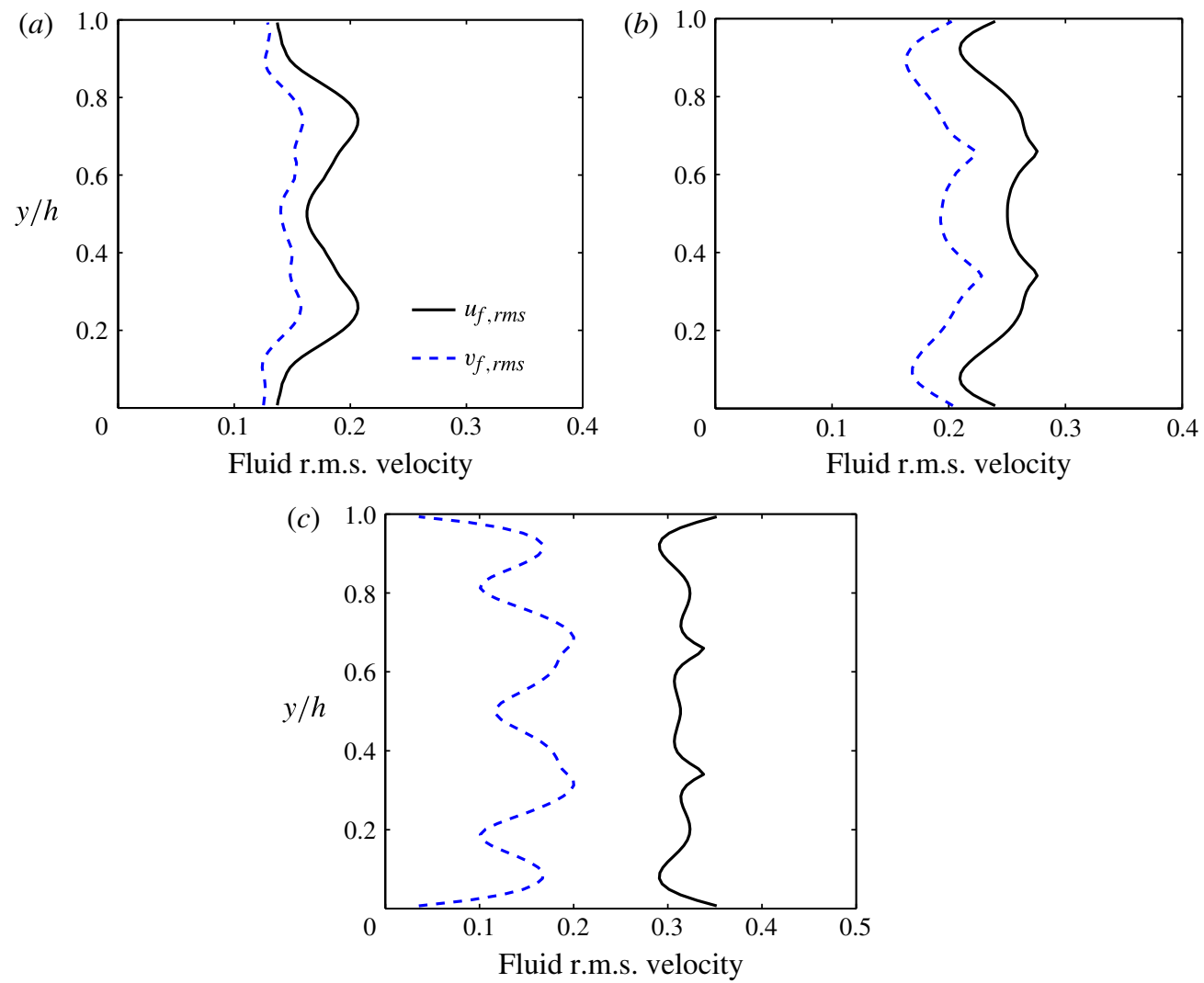

FIGURE 5. (Colour online) Vertical profile of the fluid r.m.s. velocity components, $u_{f, r m s}$ in the planar direction and $v_{f, r m s}$ in the vertical direction, in an active suspension for volume fractions of $(a) \phi=0.078,(b) \phi=0.16$ and $(c) \phi=0.24$.
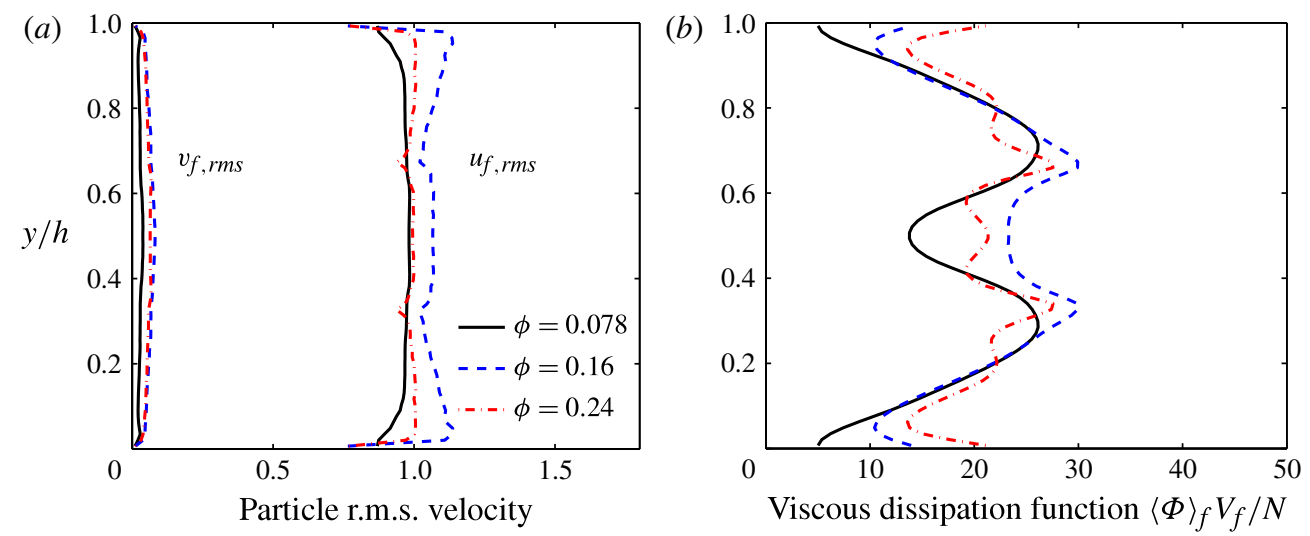

FIGURE 6. (Colour online) Vertical profiles of $(a)$ the particle r.m.s. velocity in the planar direction, $u_{p, r m s}$, and the vertical direction $v_{p, r m s}$, and $(b)$ the total viscous dissipation function, $\langle\Phi\rangle_{f} V_{f}$, normalized by the number of particles, $N$. 


\begin{tabular}{|c|c|c|}
\hline$\phi$ & $\begin{array}{l}\text { Swimming speed } \\
\left\langle u_{s}\right\rangle_{p}\end{array}$ & $\begin{array}{l}\text { Total energy per particle } \\
\qquad P=\langle\Phi\rangle_{f} V_{f} / N\end{array}$ \\
\hline 0.005 & 1.07 & 17.6 \\
\hline 0.078 & 0.97 & 17.8 \\
\hline 0.16 & 0.93 & 18.7 \\
\hline 0.24 & 0.82 & 20.2 \\
\hline
\end{tabular}

TABLE 1. The mean particle swimming speed and the total energy requirement per particle for the four volume fractions under consideration.

The vertical profile of the total mean viscous dissipation per particle, $\langle\Phi\rangle_{f} V_{f} / N$, where $V_{f}$ is the fluid volume is shown in figure $6(b)$. This is the energy for swimming generated by the particle and transferred to the fluid. The dimensionless viscous dissipation is calculated as $\Phi=2 \boldsymbol{D}: \boldsymbol{D}$ where $\boldsymbol{D}$ is the deformation tensor. The corresponding total energy requirement per particle for swimming and the mean particle swimming speed are reported in table 1 for four volume fractions, where $\phi=0.005$ corresponds to one particle inside our periodic domain. As the volume fraction increases, more energy per particle is required for the same swimming motion. The viscous dissipation per particle increases as stronger shear layers form with increasing numbers of swimming particles. The increase in the energy required by the swimming particle for increasing volume fractions corresponds to a decrease in the mean particle swimming speed, following the observations of Ishikawa \& Pedley (2007a) and Underhill et al. (2008).

\subsection{Analysis of the nutrient uptake model}

As mentioned earlier, theoretical and numerical studies of nutrient uptake typically consider a constant nutrient concentration imposed at the surface of the microorganisms. Magar et al. (2003) and Magar \& Pedley (2005) propose an alternative boundary condition for nutrient uptake that takes into account both the absorption of nutrients and nutrient diffusion inside of the cell. In this more sophisticated model, the uptake of nutrients by the swimming particle depends upon the interior nutrient concentration and it is assumed that the nutrient is consumed uniformly. Using asymptotic expansion, the governing equations are solved in the limit of very small and very large Péclet numbers. Their results show that the nutrient uptake using the alternative boundary condition behaves very differently from the case of constant nutrient concentration at the particle surface: the uptake is limited by the internal consumption when the resistance to mass transport in the fluid is very small, corresponding to large nutrient gradients in the fluid. The Sherwood number is lower than the case with constant surface concentration and this difference becomes more pronounced at very high Péclet numbers. In the analysis of an unsteady squirmer, Magar \& Pedley (2005) show that the reduction in the Sherwood number depends upon the membrane permeability and the nutrient diffusivity and identify the need for a study at intermediate values of $P e$. Before considering the case of a suspension, we wish therefore to discuss the consequences of assuming a kinetic model for the nutrient uptake and show that the swimmer mass flux is decreasing at higher $P e$, unlike the case with imposed concentration.

The difference between the nutrient uptake model of Magar et al. (2003) and the nutrient uptake model used in this study is that in the present analysis the uptake rate occurs locally at the surface of the swimming particle. The local consumption 


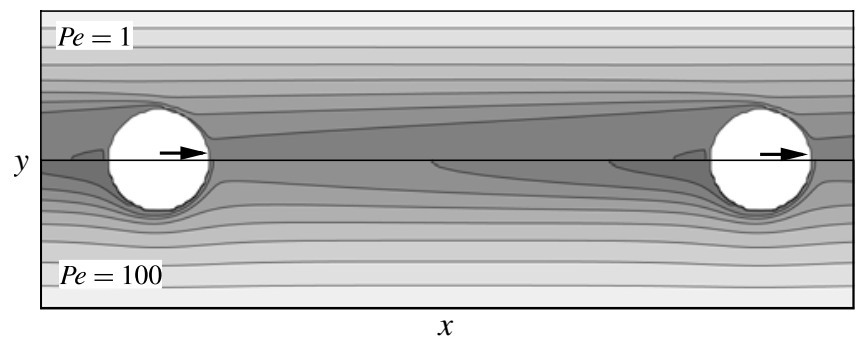

FIGURE 7. Superposition of nutrient concentration contours in a dilute suspension with $\phi=0.005$ for $P e=1$ (top frame) and $P e=100$ (bottom frame). Dark grey corresponds to $c_{f}=0$ and white to $c_{f}=1$.
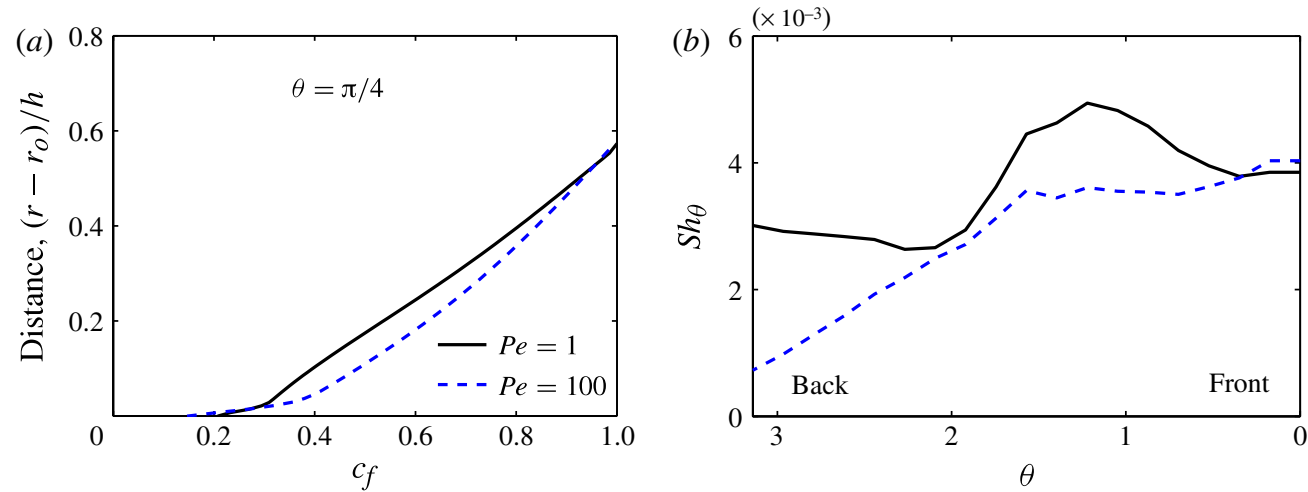

FIGURE 8. (Colour online) Comparison of $(a)$ the radial profile of the nutrient concentration, $c_{f}$, in the $x y$ plane at $\theta=\pi / 4$ and $(b)$ the local Sherwood number, $S h_{\theta}$, for changes in $P e$ with $\phi=0.005$ and $D a=1000$.

is dependent upon the nutrient concentration at the surface and is independent of the diffusion and nutrient concentrations in the particle interior. The nutrient uptake model adopted here can be seen as the limit of zero internal transport of the model proposed by Magar et al. (2003).

In order to understand how the consumption of nutrients is affected by swimming speed, nutrient concentration contours are shown in figure 7 for the case of one swimming particle translating along the thin film centreline and $P e=1$ and 100 . The figure displays the different nutrient boundary layer and nutrient wake at the back of the swimming particle at the two different swimming speeds. At $P e=1$, diffusion smooths out the concentration gradients with higher nutrient concentrations surrounding the particle surface when compared with the results for $P e=100$. At the higher swimming speed, the role of diffusion is limited and the concentration gradients in the nutrient wake are larger with lower nutrient concentrations that reach the swimming particle. To better visualize the nutrient boundary layer, we display in figure $8(a)$ the radial profiles of the nutrient concentration for the two values of $P e$ at angle $\theta=\pi / 4$ from the front of the swimming particle. The figure shows that the value of the nutrient concentration at the surface is smaller for $P e=100$ than for $P e=1$, with steeper gradients away form the surface for high $P e$. The effect of the 

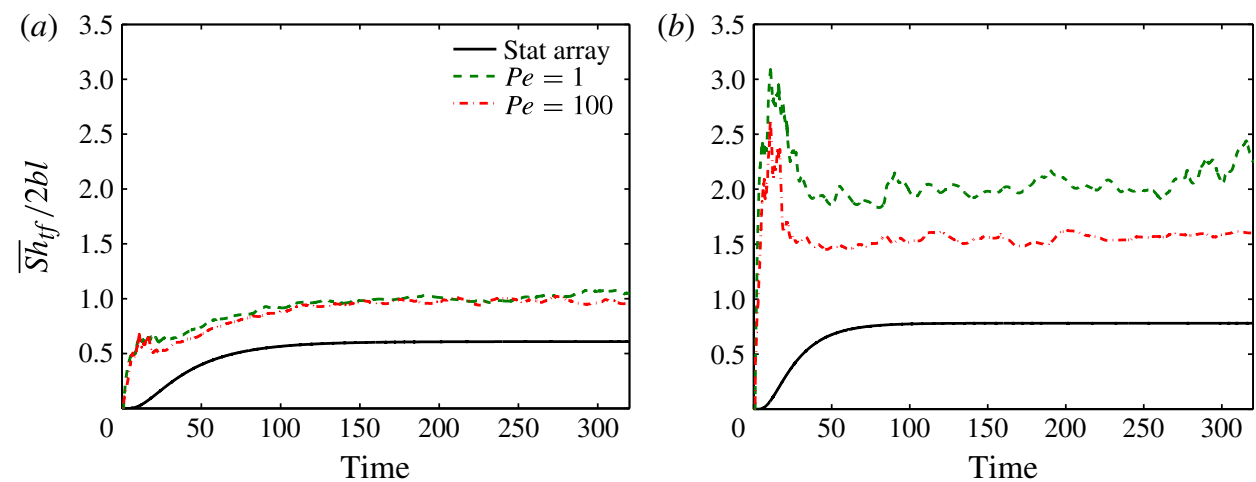

FIgURE 9. (Colour online) The time development of the overall Sherwood number, $\overline{S h}_{t f} / 2 b l$, at the thin film surface in a suspension with a volume fraction of $\phi=0.078$ for (a) $D a=100$ and (b) $D a=1000$.

lower nutrient concentrations near the particle surface on the local Sherwood number $S h_{\theta}$ is displayed in figure $8(b)$. Since the uptake of nutrients by the swimming particle is dependent upon the nutrient concentration at the surface, lower concentrations surrounding the swimming particle at $P e=100$ result in lower values of the local Sherwood number, a finding consistent with the theoretical prediction by Magar \& Pedley (2005). The results show that for an isolated squirmer, the mass flux is slightly higher at higher swimming speeds toward the front of the swimmer, with the greatest reduction in mass flux occurring toward the back of the body, in the nutrient wake region.

\subsection{Nutrient absorption and concentrations}

The mass flux at the thin film surface is created by the surface gradients that develop as the swimming and stationary particles absorb nutrients in the fluid medium. The time development of the overall Sherwood number at the thin film surface, $\overline{S h}_{t f}$, is shown in figure 9 for particle absorption rates of $D a=100$ and 1000 and a volume fraction of $\phi=0.078$. The overall $\overline{S h}_{t f}$ number is the sum of the dimensionless flux over both film surfaces divided by the total film surface area, $2 b l$. Dynamic equilibrium of the system is reached after an initial time interval in both the active and stationary cases with an interval that decreases with increasing volume fraction. The mass flux at the thin film surface increases with the particle absorption rate, $D a$, and is higher for the active suspension than for the stationary array. This confirms that swimming indeed provides a benefit in terms of nutrient uptake. The difference in the mass flux for changes in the swimming speeds, or Péclet number, become apparent at higher particle absorption rates. At $D a=1000$, the mass flux at the surface is higher for the lower swimming speed, $P e=1$, than for the higher swimming speed, $P e=100$. This is one of the main findings in our study and will be explored in greater detail later in $\S 4.4$.

The vertical profiles of the mean particle mass flux, $\langle S h\rangle_{p}$, and the mean nutrient concentration in the fluid, $\left\langle c_{f}\right\rangle$, are shown in figures 10 and 11 for two absorption rates and $\phi=0.16$. The profiles for swimming particles with $P e$ values of 1 and 100 are compared with the values for a stationary array of spherical particles. First, we observe that the profile of the mass flux in the active suspension is not uniform (see figure 10), with higher values occurring near the open surfaces that exceed the uniform mass flux 

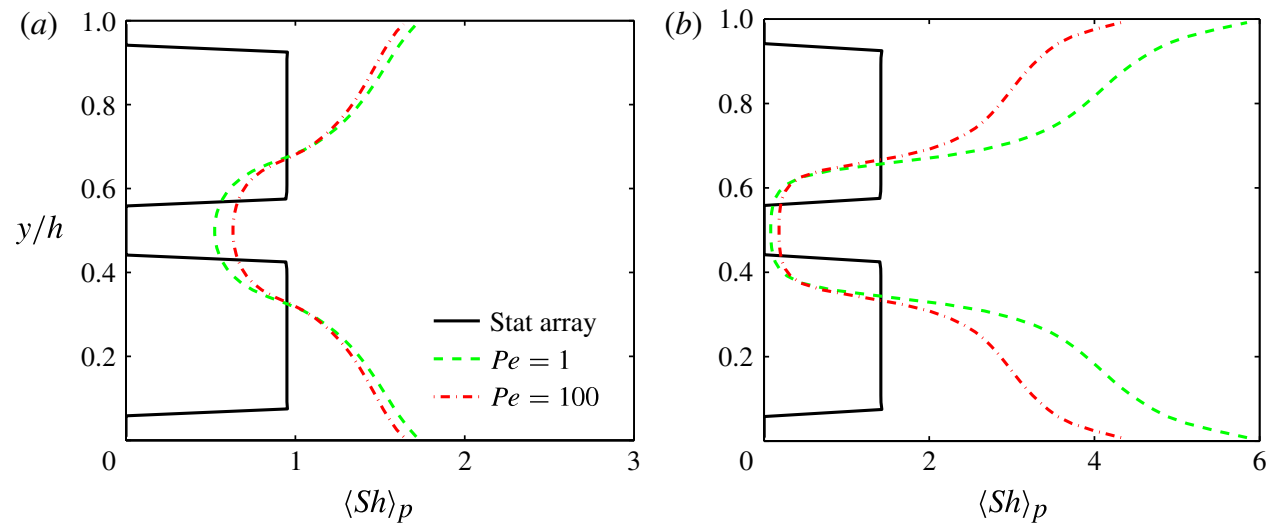

FIGURE 10. (Colour online) Vertical profiles of the mean particle Sherwood number, $\langle S h\rangle_{p}$, in a suspension with volume fraction $\phi=0.16$ for (a) $D a=100$ and (b) $D a=1000$.
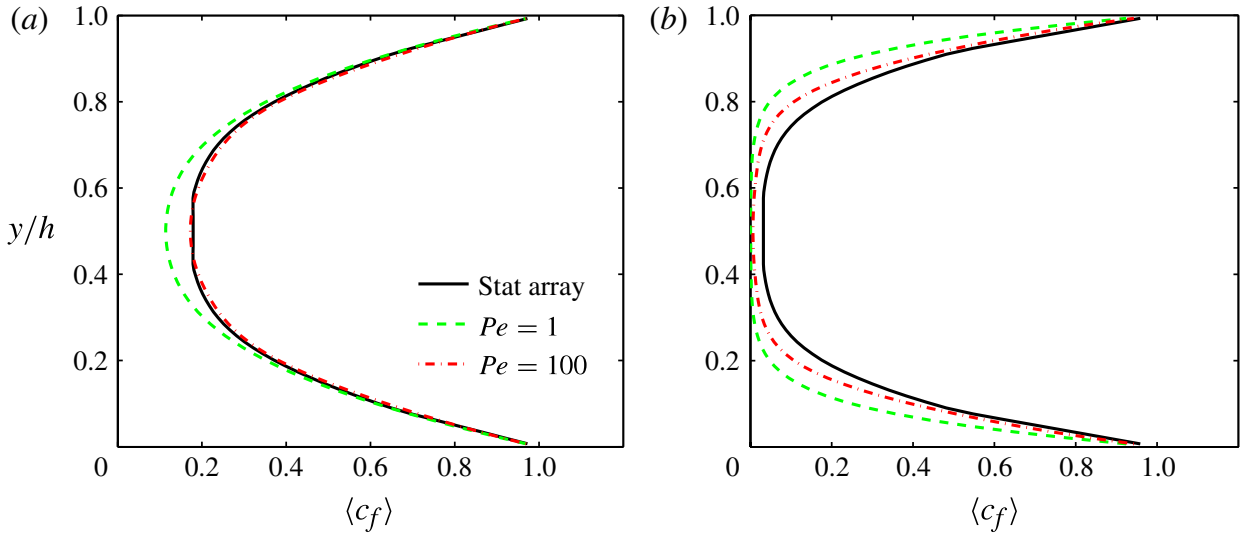

FIGURE 11. (Colour online) Vertical profiles of the mean nutrient concentration in the fluid, $\left\langle c_{f}\right\rangle$, with $\phi=0.16$ for $(a) D a=100$ and $(b) D a=1000$.

for a stationary array. The average uptake in the core region of the thin film is lower than for a stationary array. Second, the reduction in the mass flux in the core region increases for increasing absorbtion rate $D a$ as less nutrient is available in the fluid. Third, the differences in the particle uptake for changes in the swimming speed, $P e$, is more apparent at higher absorbtion rates. At $P e=1$, the mass flux in the core region is slightly lower than that for $P e=100$ whereas it is significantly higher near the open surfaces. This reduction in the spatial variation of the mass flux with increasing swimming speed is attributed to an increase in nutrient mixing throughout the film.

The effect of the absorption of nutrients on the mean concentration is shown in figure 11. The concentration profiles show that a region of nutrient depletion occurs in the film core for both swimming and stationary particles. As the absorption rate increases, the depletion zone expands in size in the vertical direction and approaches a nutrient concentration of zero. Higher nutrient concentrations are located near the film surface where the nutrients are replenished. The variation in the nutrient concentration 

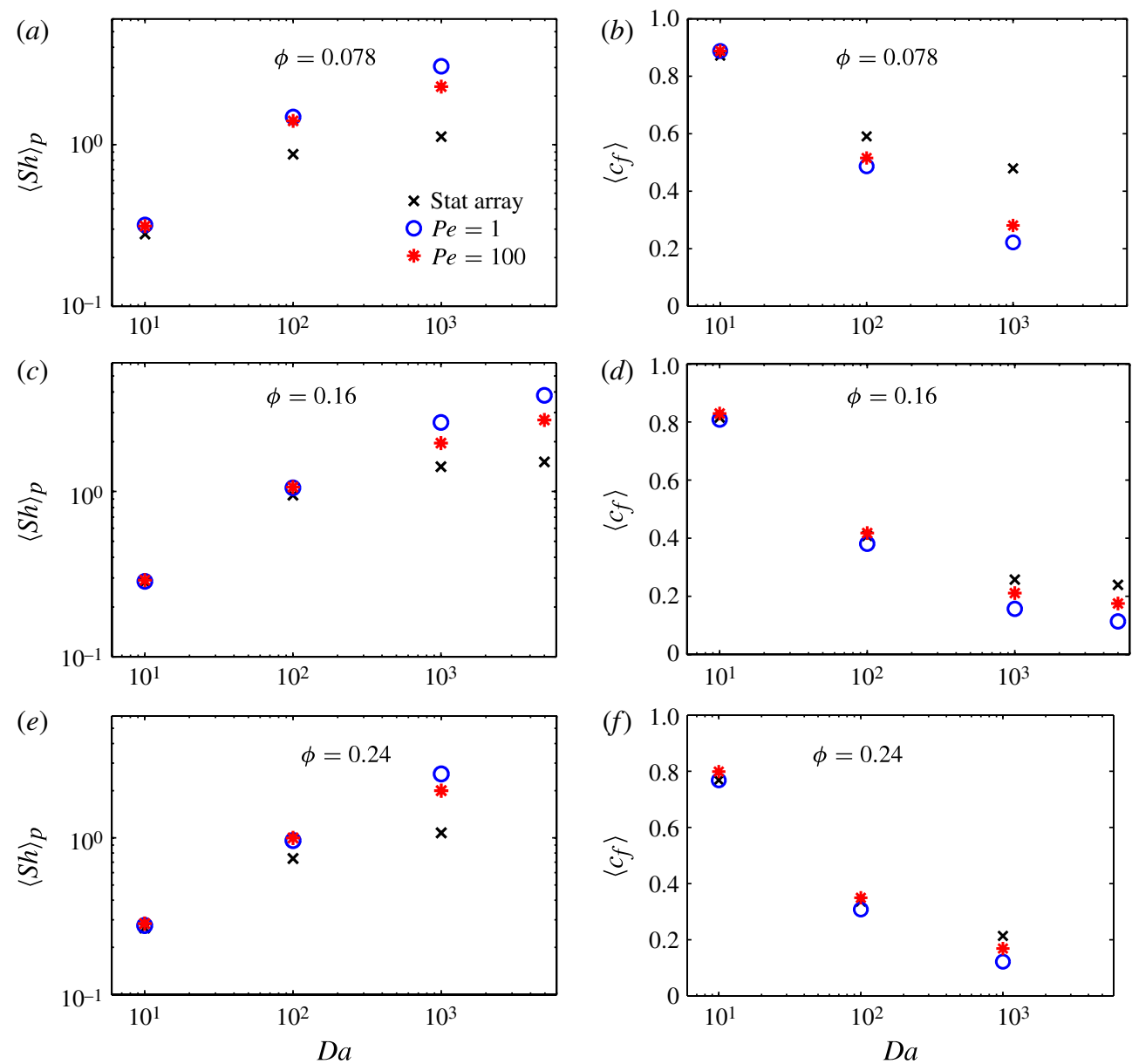

FIGURE 12. (Colour online) Ensemble averages of the $(a, c, e)$ mean particle uptake $\langle S h\rangle_{p}$ and the $(b, d, f)$ mean nutrient concentration in the fluid $\left\langle c_{f}\right\rangle$.

due to changing swimming speeds is more apparent at higher absorption rates, reflecting the variations with swimming speed discussed above: at low swimming speeds the nutrient concentrations are lower than those in the stationary case as higher mass flux is reported. At $P e=100$, the nutrient concentrations in the fluid are higher than when $P e=1$ but remain lower or equal to the nutrient concentrations observed in the stationary case.

\subsection{Ensemble averages}

The ensemble averages for the mean particle mass flux, $\langle S h\rangle_{p}$, and the fluid concentration $\left\langle c_{f}\right\rangle$, are shown in figure 12 for the investigated range in particle absorption rates, $D a$, and the three volume fractions $\phi=0.078,0.16$ and 0.24 . The results in figure $12(a-c)$ for the mean particle mass flux show that at lower absorption rates, $D a=10$, the mean uptake of the stationary and swimming particle cases are basically the same. As the absorption rate increases, the mean mass flux for the swimming particles increases, exceeding that of the stationary particles. The effect of the particle swimming motion, or the Péclet number $P e$, on the uptake becomes more 

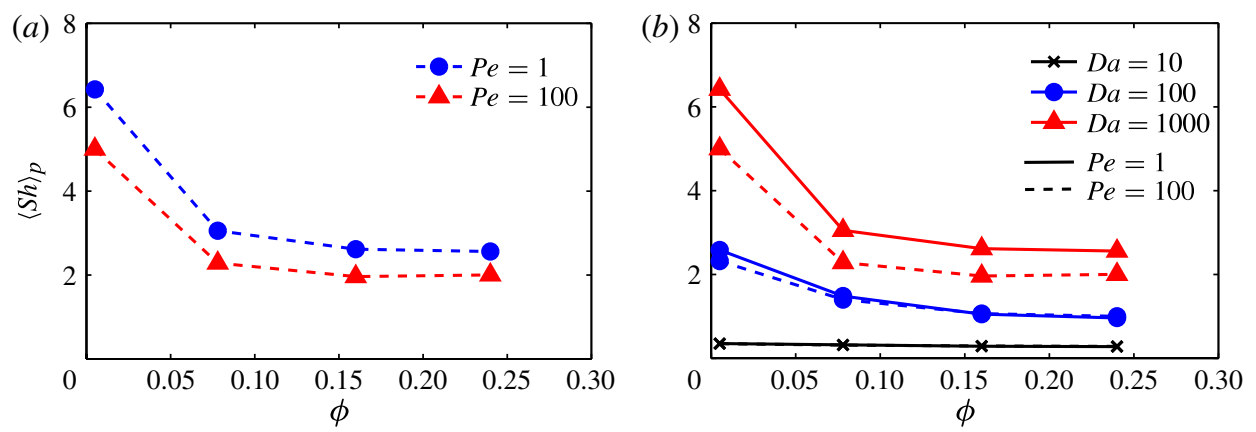

FIGURE 13. (Colour online) The variation in the mean particle mass flux, $\langle S h\rangle_{p}$, with changing volume fraction, $\phi$, for $(a) D a=1000$ and $(b) P e=1$ and 100 .

evident at higher particle absorption rates. For all cases considered, the nutrient uptake at lower swimming speeds is greater than that at higher swimming speeds, a result further discussed in figure 14.

From the results, two mass transport regimes can therefore be identified. In the first regime, for $D a \leqslant 10$, the diffusion towards the particle surface is faster than the absorption of nutrients by the particle. In this absorption rate limited regime, the swimming motion of the particles has no impact on the nutrient uptake. As the absorption rate increases, $D a \geqslant 10$, the process becomes limited by the diffusion and advection time. In this regime, the particle swimming motion enhances the particle mass flux in comparison with the stationary case. In reference to the trends in figure 12(c), test simulations in a different domain size show that as the particle absorption rate approaches infinite values, i.e. instantaneous absorption, no further increases in the particle mass flux occur. Based on this observation we anticipate that in this limit the time for absorption is much faster than the mass transport of nutrients from the bulk fluid to the particle surface. Increasing the absorption rate further has therefore no effect on the uptake at the particle surface.

The ensemble averages of the mean nutrient concentration in the fluid are given in figure $12(b, d, f)$ and show that for increasing nutrient absorption rates the nutrient concentrations in the thin film decrease. The results in the figure show that lower mean nutrient concentrations in the bulk fluid correspond to the suspensions with higher particle mass flux values. As example, since the mean particle uptake are higher for $P e=1$ than for $P e=100$, the results in the figure show that the nutrient concentrations in the fluid are lower for $P e=1$ in comparison to $P e=100$. Finally, it is important to note that the results for the three reference volume fractions show the same trends for mean nutrient uptake and nutrient concentration in the fluid for changes in $\mathrm{Pe}$ and $\mathrm{Da}$.

The influence of the volume fraction on the particle mass flux in the thin film is shown in figure $13(a, b)$ for changes in the swimming speed and the absorption rate, respectively. The results in figure 13(a) show a decrease in the particle mass flux with increasing volume fraction at a constant absorption rate of $D a=1000$. The mean particle uptake is always higher at lower values of the Péclet number for the range in volume fractions shown. We show in figure $13(b)$ that the particle uptake increases with increasing absorption rate for a fixed value of $P e$. In summary, while the particle uptake decreases with increasing volume fraction, the trends in the particle mass flux with changing $P e$ and $\mathrm{Da}$ do not vary with the volume fraction. 


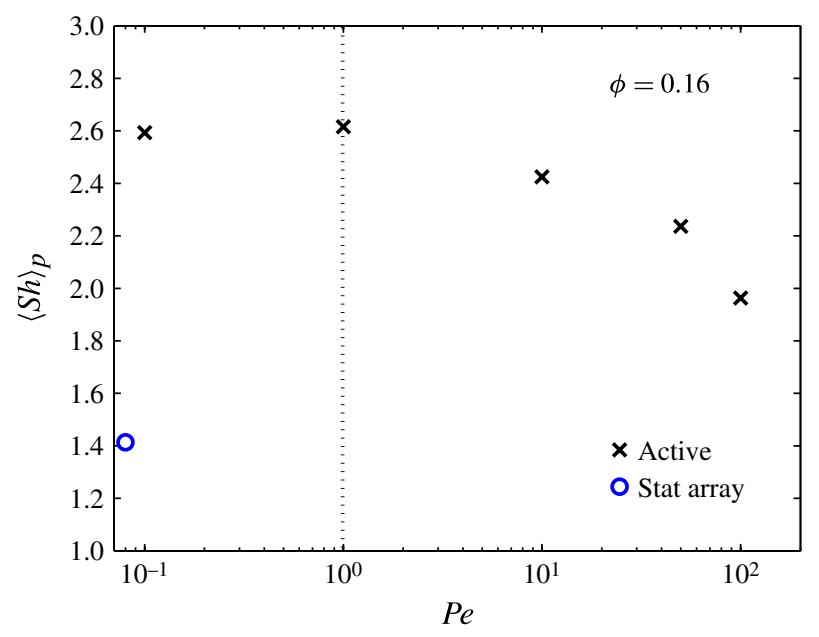

FIgURE 14. (Colour online) The variation in the mean particle mass flux, $\langle S h\rangle_{p}$, with $P e$ for $\phi=0.16$ and $D a=1000$. The open symbol reports the uptake of a stationary array of particles at the same volume fraction, the limit $P e \rightarrow 0$.

The variation in the particle mass flux with increasing swimming speed is studied via the Sherwood-Péclet relationship. This is shown in figure 14 on a log plot for a volume fraction of $\phi=0.16$ and an absorption rate of $D a=1000$. The results in the figure show that when $P e \leqslant 1$, the global particle mass flux increases with respect to that of a non-motile suspension. As the Péclet number increases above $P e=1$, the uptake decreases almost linearly. With increasing $P e$, the convection time becomes much shorter than the diffusion time and the gradients in the nutrient wake region are not diffused resulting in a larger area of lower concentration. The mechanism causing the reduction in the mass flux with increasing $P e$ was explored in $\S 4.2$ for the case of a single squirmer and attributed to lower nutrient concentrations at the swimming particle surface. In the nutrient absorption model, the consumption of nutrients is determined by the local surface concentrations which then force a local gradient along the particle surface. In the bulk fluid surrounding the particle, however, the role of diffusion is limited at higher $P e$ values and regions of lower concentrations, located primarily in the nutrient wake region, persist over a larger fluid volume having a reducing effect on the particle uptake. Comparing the data in figures 13 and 14, one can note that the differences in mean swimmer uptake observed for different $P e$ decrease as the volume fraction $\phi$ increases. This effect can be explained by the fact that the limited diffusion at higher swimming speeds is mitigated by the larger mixing induced by more frequent particle-particle interactions in a more crowded environment.

\subsection{The distribution of the particle mass flux}

The probability density function (p.d.f.), $\rho$, of the particle mass flux is shown in figure 15 for three volume fractions and an absorption rate of $D a=100$. At the lowest volume fraction, $\phi=0.078$, the particle Sherwood number $S h_{p}$ is distributed over a range in values where, for both $P e=1$ and $P e=100$, one peak in the distribution occurs and closely corresponds to the mean shown in figure $15(a)$ by vertical lines. As the volume fraction increases, the probability density as shown in figure $15(b-d)$ 
(a)

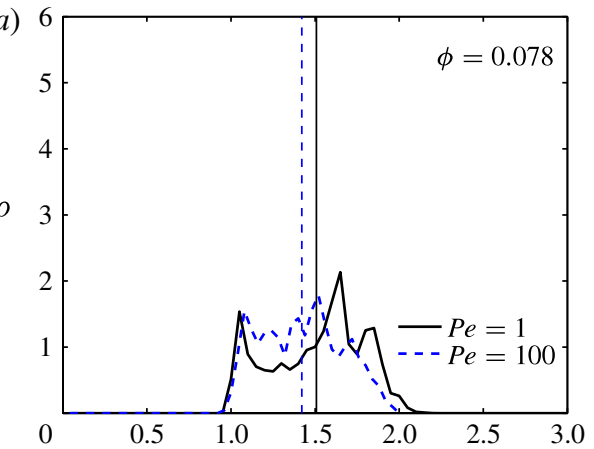

(c)

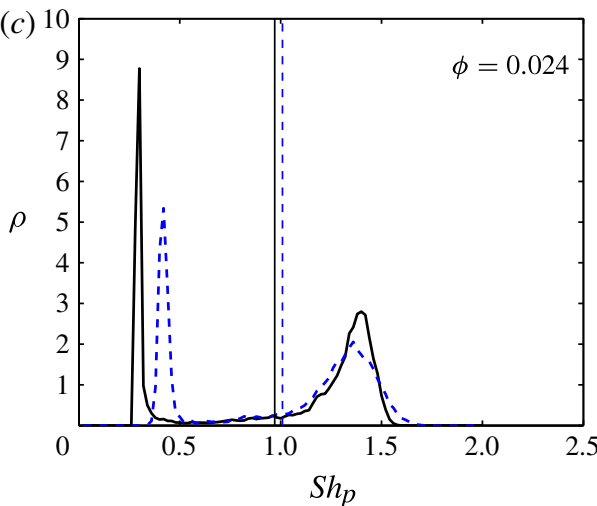

(b)

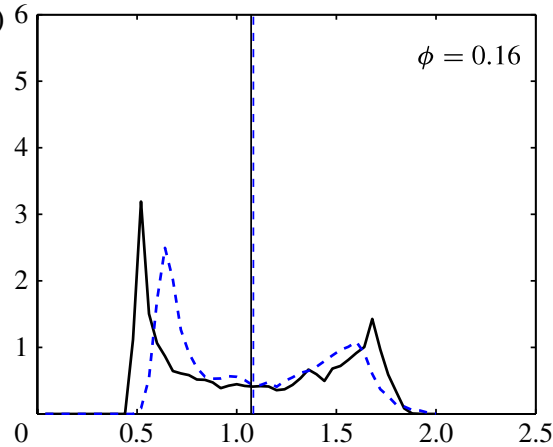

$(d)$

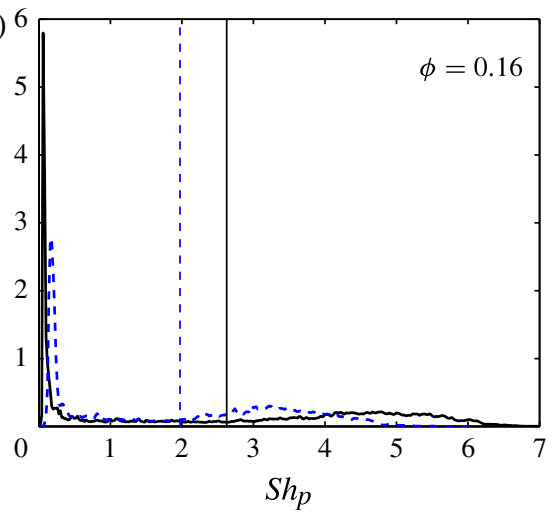

FIGURE 15. (Colour online) The p.d.f., $\rho$, of the particle mass flux, $S h_{p}$, for a nutrient absorption rate of $(a, b, c) D a=100$ and $(d) D a=1000$. The vertical lines display the mean value of each distribution.

becomes bimodal for both $P e=1$ and $P e=100$. The two peaks in the bimodal distributions are not equal, with a sharp peak in the lower $S h_{p}$ range and broader peak in the higher $S h_{p}$ range. The histogram of the mass flux p.d.f.s in the core of the thin film and in the outer fluid layers closest to the film surface, reported in figure 16, confirms that the bimodal distribution reflects the spatial variation in the mass flux observed within the thin film. The higher mass flux values occur in the fluid layers closest to the film surface whereas the lower mass flux values occur in the core region where nutrients are depleted. For increasing swimming speeds, the histogram of the particle mass flux shows that enhanced nutrient mixing results in higher particle mass flux in the thin film core.

The variance $\sigma^{2}$, the second moment of the p.d.f., $\rho$, of the particle mass flux distributions are given in table 2 for $D a=100$. The variance in the distribution function for $P e=1$ is 1.3 times larger than the variance for $P e=100$ for all three volume fractions. Furthermore, the values of $\sigma^{2}$ in the active suspension are of order $10^{-1}$ for the given absorption rate and become larger with increasing absorption rate, again showing the same trend for all three volume fractions considered. In summary, the analysis of the distribution function of the swimmer mass flux shows that nutrient uptake is intermittent in dense suspensions of swimming cells, strongly dependent on the particle position. This trend is however mitigated at higher swimming speeds: as the advection in the fluid increases, the variation in the particle mass flux decreases. 


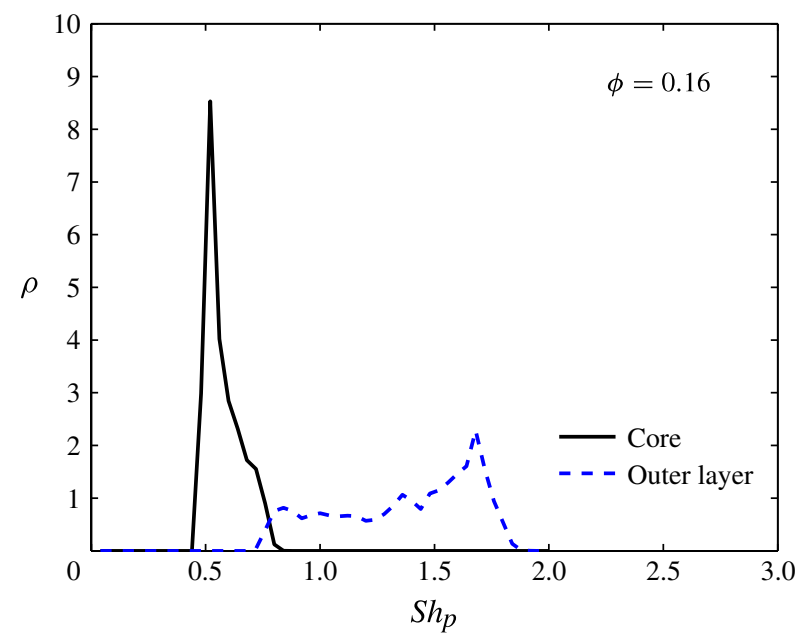

FIgURE 16. (Colour online) The spatial variation in the mass flux p.d.f., $\rho$, for $\phi=0.16$, $P e=1$ and $D a=100$.

\begin{tabular}{lccc}
\multicolumn{4}{c}{ Variance $\sigma^{2}$} \\
\hline$\phi$ & 0.078 & 0.16 & 0.24 \\
$P e=1$ & 0.077 & 0.209 & 0.240 \\
$P e=100$ & 0.058 & 0.157 & 0.188 \\
Fixed array & $2.7 \times 10^{-6}$ & $2.2 \times 10^{-5}$ & $7.4 \times 10^{-5}$
\end{tabular}

TABLE 2. Values of the variance $\sigma^{2}$ of the swimmer nutrient uptake $S h_{p}$ for $D a=100$.

We have shown in $\S 4.1$ that vertical motions are significantly suppressed in semidilute and dense suspensions and therefore squirmers can be trapped for relatively long times in regions with low nutrient concentrations. Higher swimming speeds have therefore a twofold effect: decrease the mean uptake rate by decreasing the time available for the nutrient to diffuse to the swimmer surface, see discussion of figure 14, but also create a more uniform uptake distribution among the population. The latter beneficial effect can be more significant at volume fraction higher than those considered in this study.

\section{Conclusions}

A numerical study of the mass transport in a thin film with swimming particles has been conducted using a fully resolved and novel IBM code, capable of resolving particle interactions in the concentrated suspension regime. Only a few numerical studies have been conducted for active suspensions in confined domains, with the role of hydrodynamics yet to be fully explored for the wide range in swimming types, domain sizes and swimming particle volume fractions.

The fluid flow in the thin film is generated by the swimming motion of the particles that consume nutrients in the fluid. Here neutral steady squirmers are considered. The uptake of nutrients by the swimming particles is represented by first-order kinetics applied locally at the particle surface representing an enzymatic-like process 
that captures and transforms the nutrient material inside the cellular membrane. The approach adopted in this study is in the limit of zero internal diffusion of the nutrient uptake model first proposed by Magar et al. (2003) in a mass transfer study of an individual swimming particle. This study extends previous mass transport studies of swimming micro-organisms to concentrated suspensions and to an intermediate range in both the Péclet and Damköhler numbers.

The results of the numerical study show that the vertical swimming motion in the thin film is suppressed and that the swimming particles are mainly confined to swim in the planar direction. The swimming particles have a tendency to accumulate into layers with stronger accumulation in between the film centreline and the film surface at lower volume fractions. At higher volume fractions, the preferential particle accumulation closer to the surface disappears.

We have studied how the mean nutrient uptake varies with the absorption rate, the swimming speed and the volume fraction, $S h(P e, \phi, D a)$. At lower absorption rates, the process of nutrient uptake is rate limited and particle motion in the bulk fluid has a negligible effect on the particle mass flux. As the absorption rate increases, the process of nutrient uptake is limited by the diffusion and advection time: in this regime swimming induces a significant increase in nutrient uptake as the particle mass flux is also enhanced by the swimming particle motion from regions of lower to higher nutrient concentration.

The effect of swimming particle motion on the particle mass flux becomes apparent at higher nutrient absorption rates where we show a decrease of nutrient uptake with increasing swimming speed when advection dominates the nutrient transport, $P e>1$. This effect turns out to be mainly a consequence of our first-order kinetic uptake model, assuming the mass flux proportional to the local nutrient concentration at the cell surface (see Magar \& Pedley 2005). By considering the case of very low volume fractions we demonstrate that swimmers faster than the nutrient diffusion develop sharper nutrient concentration gradients close to their body. In the nutrient wake behind the swimmers, a larger fluid volume with lower nutrient concentration develops thereby lowering the nutrient uptake at the particle surface. Since the model microorganisms tend to swim in layers in the horizontal direction, they become trapped in the advection wakes of neighbouring particles which further reduces the mass flux.

An analysis of the distribution in the particle mass flux shows a bimodal behaviour that can be attributed to significant spatial variations in uptake as opposed to temporal. The squirmers swimming through the core region of the thin film have a much lower mass flux than those moving through the fluid layers near the thin film surface. We find that these large differences between particle uptake decrease at high swimming speeds.

Finally, as the volume fraction of swimming particles in the thin film increases, more energy is required for the same swimming motion and the particle mass flux is reduced. The increase in energy required for swimming is attributed to the increase in the near-field interactions between particles whereas the decrease in the mass flux at larger volume fractions results from a higher accumulation of particles in the nutrient depletion zone in the film core. Comparing the energy loss and the uptake gain (table 1 and figures 12 and 14) it is clear that swimming is still a winning strategy for micro-organism survival in the relatively dense suspensions considered here. In the future, we aim to investigate how the response of the swimmers to the nutrient concentration (chemotaxis) as well as mechanical effects such as gyrotaxis, gravitaxis and different swimming modes alter the picture provided here. 


\section{Acknowledgements}

Computer time provided by SNIC (Swedish National Infrastructure for Computing) is gratefully acknowledged. The present work is supported by the Swedish Research Council (VR) and by the Linné Flow Centre, KTH.

\section{Appendix. Details on numerical method}

To simulate the flow and concentration field around the swimming cells we make use of an IBM (Peskin 2002; Mittal \& Iaccarino 2005). The principle of IBMs is that the cells, modelled as spheres here, are immersed in a Cartesian grid that does not conform to their shape. Instead of imposing explicit boundary conditions on the sphere/fluid interface, forces are added to the flow field in the immediate vicinity of the interface such that the boundary conditions are fulfilled to a good approximation. The IBM makes use of a fixed grid and no regridding is required for mobile particles. In addition, the Cartesian grid for the fluid phase enables the use of computationally efficient discretization schemes and flow solvers. The IBM is thus computationally efficient as compared with traditional methods which employ an unstructured, bodyfitted grid. This explains why IBMs are getting increasingly popular since the method was first introduced by Peskin in the 1970s (Peskin 1972). The challenge is to develop IBMs that are not only computationally efficient, but also sufficiently accurate.

The currently employed IBM was originally developed by Uhlmann (2005) to simulate the flow around mobile solid spheres. It has been modified by Breugem $(2010,2012)$ such that it became second-order accurate in the spatial grid resolution. The accuracy of the modified method has been demonstrated for many different flows over a range of Reynolds numbers such as the flow through an array of spheres, the drafting-kissing-tumbling interaction between two settling spheres in a closed cavity and the normal approach of two spheres at finite distance (Breugem 2012). However, since the numerical method makes use of a fixed Cartesian grid, it will eventually fail to capture the flow in the intervening film and, hence, the lubrication force between two particles just prior to a collision (Breugem 2010). For that reason lubrication force corrections have been added to the right-hand side of (2.14) when two particles are within a certain threshold distance from each other.

To determine the threshold distance at which lubrication corrections are activated, simulations have been performed of two equal spheres in shearing and in normal motion. The spheres were located in the centre of a sufficiently large computational domain with appropriate boundary conditions at the domain boundaries (Breugem 2010). The imposed translational velocity on the spheres was $u_{s}$ and $-u_{s}$, respectively, with $u_{s}=0.1 v / d$. This corresponds to a particle Reynolds number of 0.1 and the flow was thus in the Stokes regime. In the simulations the gap width between the spheres was varied. The gap width normalized with the sphere radius $R$ is denoted by $\epsilon$. The Stokes amplification factor $(\lambda)$ is defined as the drag force $\left(F_{d}\right)$ normalized by the Stokes drag force acting on a slowly moving sphere in free space $\left(6 \pi \mu R u_{s}\right)$ :

$$
\lambda(\epsilon)=F_{d} /\left(6 \pi \mu R u_{s}\right) .
$$

Figure 17(a) shows the flow field around two spheres in shearing motion at $\epsilon=0.156$ for a grid resolution of $d / \Delta x=16$. From the IBM force distribution on the Lagrangian grid of the spheres the drag force on the spheres could be determined (Breugem 2012). Figure 17(b) shows $\lambda$ as function of $\epsilon$, where each dot represents a different simulation. The simulation results appear in good agreement with the analytical solution for $\lambda$ from O'Neill (1970) for freely rotating spheres in shearing 

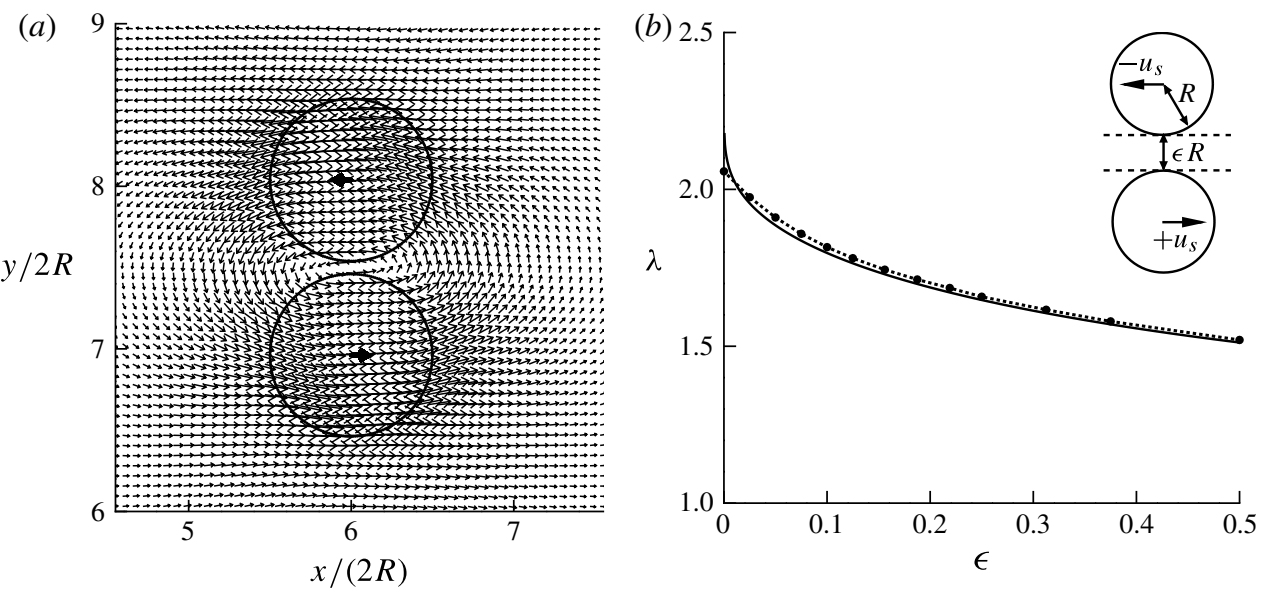

FIGURE 17. (a) Flow field around two equal solid spheres in shearing motion at $\epsilon=0.156$. The grid resolution used in the IBM simulation corresponds to $d / \Delta x=16$. (b) The Stokes amplification factor $\lambda$ as function of $\epsilon$ for two equal solid spheres in shearing motion. Dots and dotted line: results from the IBM simulations at $d / \Delta x=16$. Solid line: analytical solution from O’Neill (1970) for freely rotating spheres.

motion. The agreement is good over the whole range of $\epsilon$, even for very small values of $\epsilon$. It is therefore concluded that our numerical method does not require a lubrication correction for shearing motion at small gap widths. The drag force acting on two freely rotating spheres in parallel motion remains finite in the limit of $\epsilon \rightarrow 0$. In fact, from the exact solution it follows that at $\epsilon=0$ the angular velocity at the point of contact is exactly opposite to the translation velocity, meaning zero velocity of the point of contact and no flow in the immediate neighbourhood of this point. This can be understood from the fact that if the angular and translational velocities would not cancel each other in the point of contact, the viscous shear within the gap width would approach infinity for $\epsilon \rightarrow 0$ and this would induce a torque on the particles until the angular and translational velocities do cancel each other. The zero velocity of the point of contact is the reason why the IBM can accurately capture the Stokes amplification factor down to zero gap width; the grid resolution inside the gap does not matter in this case.

Figure 18(a) shows $\lambda$ for two equal spheres in squeezing motion for two different grid resolutions, $d / \Delta x=16$ and 32 . The simulation results are compared with the analytical solution from Brenner (1961) for this case. For $d / \Delta x=16$ the simulation results deviate from the analytical result for $\epsilon \lesssim 0.25$, which corresponds to a gap width $\lesssim 2 \Delta x$. When the grid resolution is increased to $d / \Delta x=32$ the agreement with the analytical result is good down to $\epsilon \approx 0.025$. The good agreement until $\epsilon \approx 0.025$ is quite remarkable, since it corresponds to a gap width of $\approx 0.4 \Delta x$, which is significantly smaller than the grid spacing. For smaller gap width the numerical solution is unable to capture the analytical solution, which diverges to $\infty$ for $\epsilon \rightarrow 0$. It is concluded that for spheres in squeezing motion a resolution-dependent lubrication correction is required when the gap width becomes small.

The lubrication correction for two equal spheres in squeezing motion is modelled as (Ladd \& Verberg 2001; Dance \& Maxey 2003; Breugem 2010):

$$
\boldsymbol{F}_{l c}=-6 \pi \mu R \boldsymbol{u}_{s}\left[\lambda(\epsilon)-\lambda\left(\epsilon_{l c}\right)\right],
$$



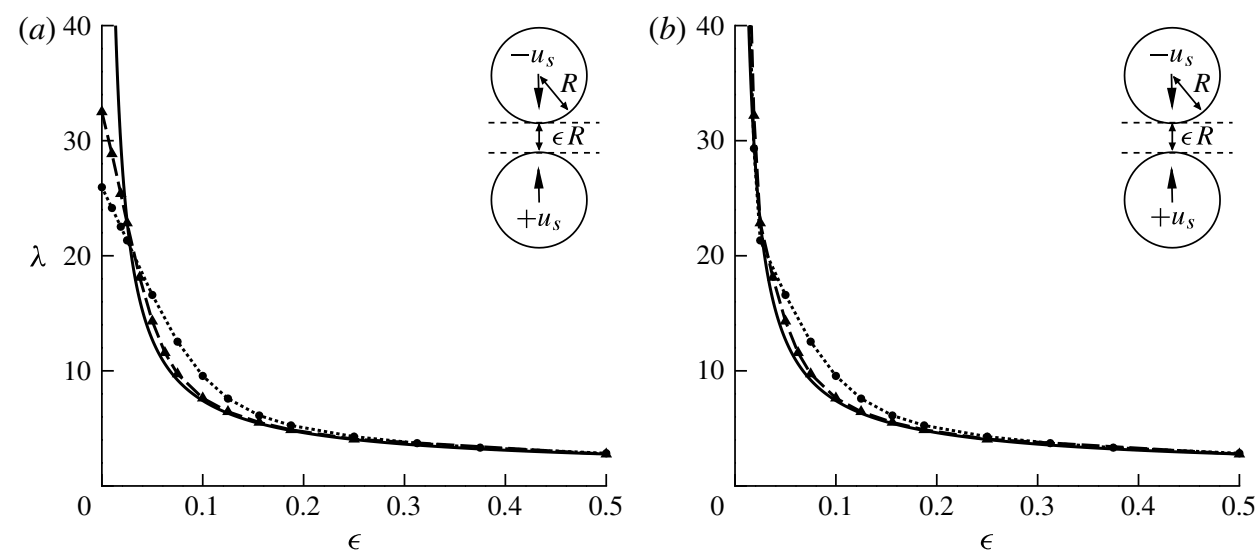

FIgURE 18. (a) The Stokes amplification factor $\lambda$ as a function of $\epsilon$ for two equal solid spheres in squeezing motion. Dots and dotted line: results from the IBM simulations at grid resolution $R / \Delta x=8$. Triangles and dashed line: results from the IBM simulation at $R / \Delta x=16$. Solid line: analytical solution from Brenner (1961). (b) Idem as in $(a)$, but now with the lubrication correction of (A $2 a$ ) for small $\epsilon$.

where $\boldsymbol{u}_{s}$ is half the relative velocity between the two spheres (along their line of centres) and $\epsilon_{l c}$ is the threshold gap width at which the lubrication correction is activated. For both $d / \Delta x=16$ and 32 we took $\epsilon_{l c}=0.025$ (the same value is used in the simulations in the present work with $d / \Delta x=24) . \lambda(\epsilon)$ is the asymptotic expansion of Brenner's analytical solution (Dance \& Maxey 2003):

$$
\lambda(\epsilon)=\frac{1}{2 \epsilon}-\frac{9}{20} \log \epsilon-\frac{3}{56} \epsilon \log \epsilon+1.346+O(\epsilon) .
$$

The lubrication force correction is added to the right-hand side of (2.14) and denoted by $f_{l c}$ (force per unit mass). Figure $18(b)$ shows the same simulation results as figure 18(a), but now including the lubrication force correction. The agreement with the analytical result is now good over the whole range of $\epsilon$, in particular for the highest resolution.

Similar to the case of spheres in shearing motion, no lubrication correction is included in our model for a sphere moving parallel to a plane wall. Based on simulations of a sphere moving normal to a plane wall, it was found that the simulation results are in good agreement with Brenner's analytical solution (Brenner 1961) for this case for $\epsilon \gtrsim 0.1$ (Breugem 2010). For $\epsilon \leqslant 0.1$ the lubrication correction is modelled similar to (A $2 a$ ) with the expression for $\lambda$ equal to the asymptotic expansion of Brenner's analytical solution (Jeffrey 1982; Breugem 2010):

$$
\lambda(\epsilon)=\frac{1}{\epsilon}-\frac{1}{5} \log \epsilon-\frac{1}{21} \epsilon \log \epsilon+0.9713+O(\epsilon) .
$$

The asymptotic expansions of $\lambda$ for two spheres in squeezing motion and for the head-on collision of a sphere onto a plane wall diverge to $\infty$ for $\epsilon \rightarrow 0$. This will actually prevent any collision. Note, however, that the asymptotic expansions have been derived for a perfectly smooth and inelastic sphere/wall and are based on the assumption that the Stokes equations still hold for the flow in the intervening film when $\epsilon \rightarrow 0$. From experiments it is known that surface roughness elements of the sphere/wall cause actual sphere-sphere/wall contact when the gap width $(\epsilon R)$ is of 


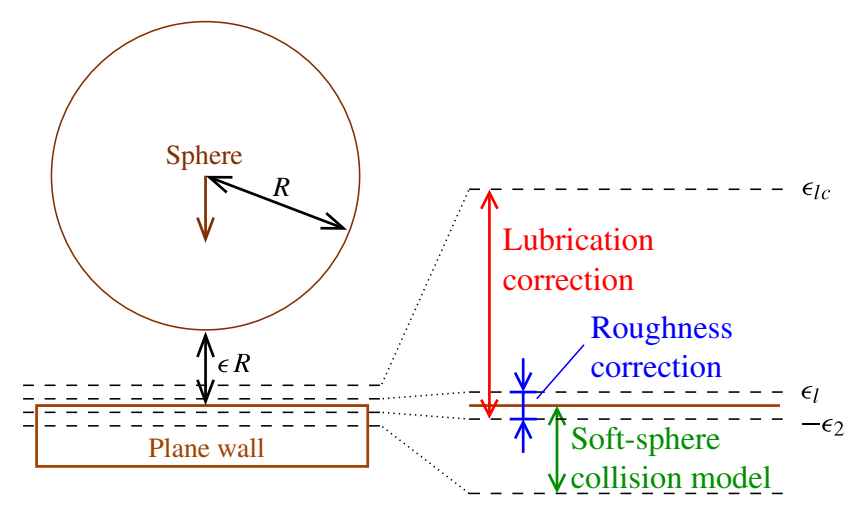

FIGURE 19. (Colour online) Illustration of the soft-sphere collision model combined with a lubrication correction for the head-on collision of a sphere onto a plane wall. The parameters $\epsilon_{l c}, \epsilon_{1}$ and $\epsilon_{2}$ are explained in the text.

the order of the typical size of the surface roughness elements (Joseph et al. 2006). This will hamper further drainage of the intervening film and the drainage process will eventually stop when many roughness elements have made contact with each other. This effect is modelled by fixing the lubrication correction at $\lambda(\epsilon)=\lambda\left(\epsilon_{1}\right)$ for $-\epsilon_{2} \leqslant \epsilon \leqslant \epsilon_{1}$, where $\epsilon_{1}=1 \times 10^{-3}$ and $\epsilon_{2}=1 \times 10^{-2}$ are empirical constants. For $\epsilon \leqslant-\epsilon_{2}$ the lubrication force correction is turned off.

The lubrication correction model is combined with a soft-sphere collision model (Van der Hoef et al. 2006) to compute the repulsive force between particles once solid contact is made $(\epsilon \leqslant 0)$. This is illustrated in figure 19 for a sphere colliding onto a plane wall. In the soft-sphere collision model particles are allowed to slightly overlap with each other. The solid contact force is computed from the overlap between the particles and their relative velocity. The collision force is decomposed into a normal and tangential component, where the normal component is parallel to the unit normal on the particle interface at the point of particle contact. In the present study only the normal contact force was taken into account. The normal component of the contact force acting on particle $a$ when in contact with particle $b$ is parameterized as

$$
\boldsymbol{F}_{r}=-k \delta \boldsymbol{n}_{a b}-\eta\left(\boldsymbol{u}_{a b} \cdot \boldsymbol{n}_{a b}\right) \boldsymbol{n}_{a b},
$$

where $k$ is the spring stiffness, $\eta$ is the damping coefficient, $\delta$ is the overlap between the particles, $\boldsymbol{n}_{a b}$ is the unit normal and $\boldsymbol{u}_{a b}$ is the relative velocity between the particles. Input parameters of the soft-sphere collision model are the dry coefficient of restitution (for collisions in vacuum), $e_{d}$, and the time duration of a collision given as the number, $N_{c}$, of computational time steps, $\Delta t$. The collision time can be artificially stretched and is chosen much larger than predicted by Hertz contact theory (Johnson 1985) to avoid severe constraints on the numerical time step. The simulations in the present work are based on $N_{c}=8$. For more details on the collision model the reader is referred to Breugem (2010). The repulsive collision force is added to the right-hand side of (2.14) and denoted by $\boldsymbol{f}_{r}$ (force per unit mass).

The collision model combined with the lubrication correction model is illustrated in figure 19 for a sphere colliding onto a plane wall. The overall numerical method (collision model + lubrication correction model + IBM) has been thoroughly tested. The constants $\epsilon_{1}$ and $\epsilon_{2}$ have been systematically varied in a large number of simulations of a solid sphere colliding onto a plane wall in a closed cavity filled 
with a viscous fluid. The chosen values of $\epsilon_{1}$ and $\epsilon_{2}$ yielded a good agreement with the experimentally observed behaviour of the coefficient of restitution as function of the impact Stokes number (Legendre, Daniel \& Guiraud 2005; Breugem 2010).

\section{REFERENCES}

Berke, A. P., Turner, L., Berg, H. C. \& Lauga, E. 2008 Hydrodynamic attraction of swimming microorganisms by surfaces. Phys. Rev. Lett. 101, 038102.

Blake, J. R. 1971 A spherical envelope approach to ciliary propulsion. J. Fluid Mech. 46, 199-208.

BRENNER, H. 1961 The slow motion of a sphere through a viscous fluid towards a plane surface. Chem. Engng Sci. 16, 242-251.

BREUGEM, W.-P. 2010 A combined soft-sphere collision/immersed boundary method for resolved simulations of particulate flows. In Proceedings of the ASME $20103 \mathrm{rd}$ Joint US-European Fluids Engineering Summer Meeting and 8th International Conference on Nanochannels, Microchannels, and Minichannels, Montreal, Canada, p. 11.

BREUGEM, W.-P. 2012 A second-order accurate immersed boundary method for fully resolved simulations of particle-laden flows. J. Comput. Phys. 231 (13), 4469-4498.

DANCE, S. L. \& MAXEY, M. R. 2003 Incorporation of lubrication effects into the force-coupling method for particulate two-phase flows. J. Comput. Phys. 189, 212-238.

Doostmohammadi, A., Stocker, R. \& Ardekani, A. M. 2012 Low-Reynolds-number swimming at pycnoclines. Proc. Natl Acad. Sci. USA 109 (10), 3856-3861.

Downton, M. T. \& STARK, H. 2009 Simulation of a model microswimmer. J. Phys.: Condens. Matter 21, 204101.

Evans, A. A., Ishikawa, T., Yamaguchi, T. \& Lauga, E. 2011 Orientational order in concentrated suspensions of spherical microswimmers. Phys. Fluids 23 (11), 111702.

Hernandez-Ortiz, J. P., Stoltz, C. G. \& Graham, M. D. 2005 Transport and collective dynamics in suspensions of confined swimming particles. Phys. Rev. Lett. 95, 204501.

Van der Hoef, M. A., Ye, M., Van Sint Annaland, M., Andrews, A. T. IV, Sundaresan, S. \& Kuipers, J. A. M. 2006 Multi-scale modelling of gas-fluidized beds. Adv. Chem. Engng 31, 65-149.

IshikawA, T., Locsei, J. T. \& Pedley, T. J. 2008 Development of coherent structures in concentrated suspensions of swimming model micro-organisms. J. Fluid Mech. 615, 401-431.

Ishikawa, T., Locsei, J. T. \& Pedley, T. J. 2010 Fluid particle diffusion in a semidilute suspension of model micro-organisms. Phys. Rev. E 82 (2), 021408.

Ishikawa, T. \& Pedley, T. J. 2007a Diffusion of swimming model micro-organisms in a semi-dilute suspension. J. Fluid Mech. 588, 437-462.

Ishikawa, T. \& Pedley, T. J. 2007b The rheology of a semi-dilute suspension of swimming model micro-organisms. J. Fluid Mech. 588, 399-435.

IshikaWA, T. \& PeDley, T. J. 2008 Coherent structures in monolayers of swimming particles. Phys. Rev. Lett. 100 (8), 088103.

Ishikawa, T., Simmonds, M. P. \& Pedley, T. J. 2006 Hydrodynamic interaction of two swimming model micro-organisms. J. Fluid Mech. 568, 119-160.

Ishikawa, T., Yoshida, N., Ueno, H., Wiedeman, M., Imai, Y. \& Yamaguchi, T. 2011 Energy transport in a concentrated suspension of bacteria. Phys. Rev. Lett. 107, 028102.

JEFFREY, D. J. 1982 Low-Reynolds-number flow between converging spheres. Mathematika 29, $58-66$.

Johnson, K. L. 1985 Contact Mechanics. Cambridge University Press.

Joseph, G. G., Zenit, R., Hunt, M. L. \& Rosenwinkel, A. M. 2006 Particle-wall collisions in a viscous fluid. J. Fluid Mech. 443, 329-346.

Koch, D. L. \& Subramanian, G. 2011 Collective hydrodynamics of swimming microorganisms: living fluids. Annu. Rev. Fluid Mech. 43, 637-659.

Kurtuldu, H., Guasto, J. S., Johnson, K. A. \& Gollub, J. P. 2011 Enhancement of biomixing by swimming algal cells in two-dimensional films. Proc. Natl Acad. Sci. USA 108 (26), 10391-10395. 
LAdD, A. J. C. \& Verberg, R. 2001 Lattice-Boltzmann simulations of particle-fluid suspensions. J. Stat. Phys. 104, 1191-1251.

Legendre, D., Daniel, C. \& Guiraud, P. 2005 Experimental study of a drop bouncing on a wall in a liquid. Phys. Fluids 17 (097105).

Li, G., Bensson, J., Nisimova, L., Munger, D., Mahautmr, P., Tang, J. X., Maxey, M. R. \& BRUN, Y. V. 2011 Accumulation of swimming bacteria near a solid surface. Phys. Rev. E 84 (4), 041932.

Lighthill, M. J. 1952 On the squirming motion of nearly spherical deformable bodies through liquids at very small Reynolds numbers. Commun. Pure Appl. Maths 5, 109-118.

Lin, Z., Thiffeault, J.-L. \& Childress, S. 2011 Stirring by squirmers. J. Fluid Mech. 669, $167-177$.

Magar, V., Goto, T. \& Pedley, T. J. 2003 Nutrient uptake by a self-propelled steady squirmer. Q. J. Mech. Appl. Math 56 (1), 65-91.

Magar, V. \& Pedley, T. J. 2005 Average nutrient uptake by a self-propelled unsteady squirmer. J. Fluid Mech. 539, 93-112.

Michelin, S. \& LAUGA, E. 2011 Optimal feeding is optimal swimming for all Péclet numbers. Phys. Fluids 23 (10), 101901.

Mittal, R. \& IAcCARino, G. 2005 Immersed boundary methods. Annu. Rev. Fluid Mech. 37, 239-261.

Musielak, M. M., Karp-Boss, L., Jumars, P. A. \& Fauci, L. J. 2009 Nutrient transport and acquisition by diatom chains in a moving fluid. J. Fluid Mech. 638, 401-421.

O'NeILL, M. E. 1970 Exact solutions of the equations of slow viscous flow generated by the asymmetrical motion of two equal spheres. Appl. Sci. Res. 21, 452-465.

Pedley, T. J. \& Kessler, J. O. 1992 Hydrodynamic phenomena in suspensions of swimming microorganisms. Annu. Rev. Fluid Mech. 24 (1), 313-358.

Peskin, C. S. 1972 Flow patterns around heart valves: a numerical method. J. Comput. Phys. 10 (2), 252-271.

Peskin, C. S. 2002 The immersed boundary method. Acta Numerica 11, 479-517.

Saintillan, D. \& Shelley, M. J. 2007 Orientational order and instabilities in suspensions of self-locomoting rods. Phys. Rev. Lett. 99, 058102.

Short, M. B., Solari, C. A., Ganguly, S., Powers, T. R., Kessler, J. O. \& Goldstein, R. E. 2006 Flows driven by flagella of multicellular organisms enhance long-range molecular transport. Proc. Natl Acad. Sci. USA 103 (22), 8315-8319.

Spagnolie, S. E. \& LAUGA, E. 2012 Hydrodynamics of self-propulsion near a boundary: predictions and accuracy of far-field approximations. J. Fluid Mech. (submitted).

Taherzadeh, D., Picioreanu, C. \& Horn, H. 2012 Mass transfer enhcancement in moving biofilm structures. Biophys. J. 102 (7), 1483-1492.

Uhlmann, M. 2005 An immersed boundary method with direct forcing for simulation of particulate flows. J. Comput. Phys. 209, 448-476.

Underhill, P. T., Hernandez-Ortiz, J. P. \& Graham, M. D. 2008 Diffusion and spatial correlations in suspensions of swimming particles. Phys. Rev. Lett. 100 (24), 248101.

Wu, X.-L. \& LIBCHABER, A. 2000 Particle diffusion in a quasi-two-dimensional bacterial bath. Phys. Rev. Lett. 84, 3017-3020.

YEO, K. \& MAXEY, M. R. 2010 Dynamics of concentrated suspensions of non-colloidal particles in couette flow. J. Fluid Mech. 649, 205-231.

Zhu, L., Do-Quang, M., LAUga, E. \& Brandt, L. 2011 Locomotion by tangential deformation in a polynumeric fluid. Phys. Rev. E 83, 011901.

Zhu, L., LAUGa, E. \& BRANDT, L. 2012 Self-propulsion in viscoelastic fluids: pushers vs. pullers. Phys. Fluids 24, 051902.

ZhU, L., LAUGA, E. \& BRANDT, L. 2013 Low-Reynolds number swimming in a capillary tube. J. Fluid Mech. 726, 285-311. 